\title{
Spinal dI 2 interneurons regulate the stability of bipedal stepping
}

2

3 Baruch Haimson ${ }^{1}$, Yoav Hadas ${ }^{1}$, Artur Kania ${ }^{2}$, Monica Daley ${ }^{3}$, Yuval Cinnamon ${ }^{4}$, Aharon Lev-

$4 \operatorname{Tov}^{1,{ }^{*}}$ and Avihu Klar ${ }^{1, *}$

5

6

1 - Department of Medical Neurobiology, IMRIC, Hebrew University-Hadassah Medical School, Jerusalem, 91120, Israel

2 - Institut de recherches cliniques de Montréal (IRCM), Montréal, QC, H2W 1R7, Canada

3 - Ecology and Evolutionary Biology, University of California, Irvine, CA, USA

4 - Institute of Animal Science Poultry and Aquaculture Sci. Dept. Agricultural Research Organization, The Volcani Center, Israel

\section{* - Corresponding authors}

Avihu Klar

Department of medical Neurobiology

Hadassah Medical School

The Hebrew University, Jerusalem

91120

Israel

avihu.klar@mail.huji.ac.il

\section{Aharon Lev-Tov}

Department of medical Neurobiology

Hadassah Medical School

The Hebrew University, Jerusalem

91120

Israel

aharonl@ekmd.huji.ac.il 


\section{Summary}

31 Peripheral and intraspinal feedback is required to shape and update the output of spinal networks that execute motor behavior. We report that lumbar dl2 spinal interneurons of the chick receive synaptic input from afferents and pre-motoneurons. They innervate contralateral premotor networks in the lumbar and brachial spinal cord and their ascending projections innervate the cerebellum. These findings suggest that dI2 neurons function as interneurons in local lumbar circuits and involved in lumbo-brachial coupling and that part of them deliver peripheral and intraspinal feedback to the cerebellum. Silencing of dI2 neurons leads to destabilized stepping in P8 hatchlings with occasional collapses, variable stepprofiles and wide-base walking, suggesting that the dI2 neurons may contribute to stabilization of the bipedal gait.

Keywords: spinal cord, spinocerebellar tract, neuronal circuits, locomotion 


\section{Introduction}

51 The spinal cord integrates and relays the somatosensory inputs required for further

execution of complex motor behaviors. Interneurons that differentiate at the ventral progenitor domain, $\mathrm{V} 3-\mathrm{V} 0$, are involved in the control of rhythmic motor activity, alternating between left and right limbs, as well as between flexor and extensor muscles (Lai et al., 2016, Osseward and Pfaff, 2019, Alaynick et al., 2011). Some of the dorsally born interneurons, $\mathrm{dl} 1, \mathrm{dl} 3$ and dl6, migrate ventrally and are also assembled within circuitry that control motor activity (Yuengert et al., 2015, Bui et al., 2013, Andersson et al., 2012), while other dorsal progenitor neurons, $\mathrm{d} / 4$ and $\mathrm{dl} 5$, give rise to interneurons that mediate somatosensation (Lai et al., 2016). The role of dI2 neuron is elusive due to the lack of genetic targeting means. Employing intersectional genetics in the chick spinal cord we targeted dl2 and present evidences that implicate them in the control of stability during locomotion. The maintenance of stability of the body and the coordination, precision and timing of movements are regulated and modulated by the cerebellum. Anatomical and electrophysiological studies of cats and rodents revealed two major pathways ascending from tract neurons in the lumbar spinal cord to the cerebellum: the dorsal and the ventral spinocerebellar tract (DSCT and VSCT). DSCT tract neurons are considered to relay mainly proprioceptive information, while VSCT tract neurons are thought to relay internal spinal network-information to the cerebellum in addition to proprioceptive data (Jankowska and Hammar, 2013, Spanne and Jorntell, 2013, Stecina et al., 2013, Jiang et al., 2015). While subpopulations of DSCT neuron are genetically accessible (Hantman and Jessell, 2010), the genetic inaccessibility of VSCT neurons hinders revealing their actual contribution to the regulatory functions of the cerebellum in locomotion and other motor behaviors. 
In the present study we studied the possible functions of $\mathrm{dl} 2$ neurons in chick motor

74 behavior. There are several reasons and advantages to perform these studies in chicks: The

75 patterning of neurons within the spinal cord (Jessell, 2000) and the spinocerebellar tracts

76 (Furue et al., 2010, Furue et al., 2011, Uehara et al., 2012) are conserved between

77 mammalians and avian. In addition, chick uses bipedal locomotion (evolved in humans and

78 birds) that can be examined soon after hatching. For decoding the circuitry and function of

79 spinal interneurons we developed a unique circuit-deciphering toolbox that enables

80 neuronal-specific targeting and tracing of circuits in the chick embryo (Hadas et al., 2014),

81 and utilized kinematic analysis of overground bipedal stepping of the hatched chicks

82 following silencing of d 12 .

Our studies revealed that lumbar dl2 neurons receive synaptic inputs from inhibitory and excitatory premotoneurons and relay output to the cerebellar granular layer, premotor neurons at the contralateral spinal cord and the contralateral dI2. Kinematic analysis of overground stepping of P8 hatchlings after Inhibition of the neuronal activity of dI2s by targeted expression of the tetanus toxin gene, showed unstable stepping in the genetically manipulated hatchlings, hence demonstrating that dI2s play a role in shaping and stabilizing the bipedal gait. 


\section{Results}

In order to define potential VSCT neurons within spinal interneurons, we set the following criteria: 1) Soma location in accordance with pre-cerebellar neurons at the lumbar level, which were previously revealed by retrograde labeling experiments of the chick cerebellar lobes (Furue et al., 2010, Furue et al., 2011, Uehara et al., 2012), 2) Commissural neurons, 3) excitatory neurons, and 4) non-premotoneurons (Lai et al., 2016, Osseward and Pfaff, 2019, Alaynick et al., 2011). Based on these criteria dI1c and dI2 neurons are likely candidates (Bermingham et al., 2001, Yuengert et al., 2015) (Fig. S1B). This is further supported by Sakai et al., who demonstrated that in the E12 chick, dI1 and dI2 axons project to the hindbrain and toward the cerebellum (Sakai et al., 2012). In the current study we have focused on deciphering the circuitry and function of dI2 neurons and their possible association to VSCT.

\section{dI2 interneurons are mainly excitatory neurons with commissural axonal projection}

The dI2 neurons originate at the dorsal spinal cord. The progenitor pdI2 express Ngn1, Ngn2, Olig3 and Pax3 transcription factors (TFs). The post mitotic dI2 are defined by a combinatorial expression of Foxd3 ${ }^{+} /$Lhx $1^{+} /$Pou $4 f 1^{+}$TFs (Alaynick et al., 2011, Morikawa et al., 2009, Francius et al., 2013). To label dI2 neurons, axons, and terminals in the chick spinal cord we used intersection between enhancers of two dI2's TFs - Ngn1 and the Foxd3, via expression of two recombinases (Cre and FLPo) and double conditional reporters (Fig. S1A).

We have shown previously that the combination of these enhancers reliably labels $\mathrm{d} / 2$ neurons (Avraham et al., 2009, Hadas et al., 2014). At E5, early post mitotic dI2 neurons migrate ventrally from the dorso-lateral to the mid-lateral spinal cord (Fig. 1A). As they migrate ventrally, at E6, $\mathrm{dl} 2$ neurons assume a mid-lateral position along the dorso-ventral axis (Fig. 1B). Subsequently, dI2 neurons migrate medially, and at E17, comparable to pos- 
114 gestation day 4 (P4) of mouse, they occupy lamina VII (Fig. 1C). At all rostro-caudal levels and

115 embryonic stages, dI2 axons cross the floor plate (Fig. 1A-D). Post-crossing dI2 axons extend

116 rostrally for a few segments at the ventral funiculus (VF) and subsequently turn into the

117 lateral funiculus (LF) (Avraham et al., 2009) (Fig. 1C). The VF to LF rerouting is apparent at

118 the rostral thoracic levels (Fig. 1D). Collaterals originating from the crossed VF and LF tracts

119 invade the contralateral spinal cord (Fig. 1C, D, Fig. 4A,B).

A recent study suggested that $\mathrm{dl} 2$ neurons at early stages of development in mice at

121 E9.5-E13.5 (comparable the chick E4-8) can be divided into several sub classes based on their

122 genetic signature, and degree of maturation (Delile et al., 2019). To assess the diversity of

$123 \mathrm{~d}$ I2 neurons in the chick, the expression of dI2 TFs in dI2::GFP cells was analyzed at E5 before

124 and during ventral migration, E6 and E14. The early post mitotic dI2::GFP at E5 are a

125 homogenous population defined by Foxd $3^{+} /$Lhx1 $1^{+} /$Pou $4 f 1^{+} /$Pax2 (Fig. 1E, S2A,B). The dI2

126 neurons that undergo ventral migration at $\mathrm{E} 5$, as well as at $\mathrm{E} 6$ and $\mathrm{E} 14$, express variable

127 combinations of Lhx1, Pou4f1 and FoxP1/2/4 (Fig. 1E, S2C,D). At E14, about 50\% of dI2::GFP

128 did not express any of the tested TFs (Fig. 1E), suggesting that the early expression of TFs is

129 required for cell fate acquisition, axon guidance, and target recognition, while their

130 expression is redundant during circuitry formation, as shown for other spinal INs (Bikoff et

131 al., 2016). Interestingly, about $12 \%$ of ventrally migrating dI2 neurons (from E5 to E14)

132 express Pax2 (Fig. 1E, S2D). Pax2 is associated with an inhibitory phenotype (Cheng et al.,

133 2004), suggesting that a subpopulation of dl2 are inhibitory neurons. The distribution of

134 excitatory and inhibitory dI2 neurons is also apparent at E17. In situ hybridization on cross

135 sections of E17 dI2::GFP-labeled lumbar spinal cord, the vGlut2 probe revealed that $88 \%$

136 were vGlut2 ${ }^{+}$, while the Gad1 probe measured $25 \%$ Gad1 ${ }^{+}$dI2 neurons (Fig. S3A-D). A similar 

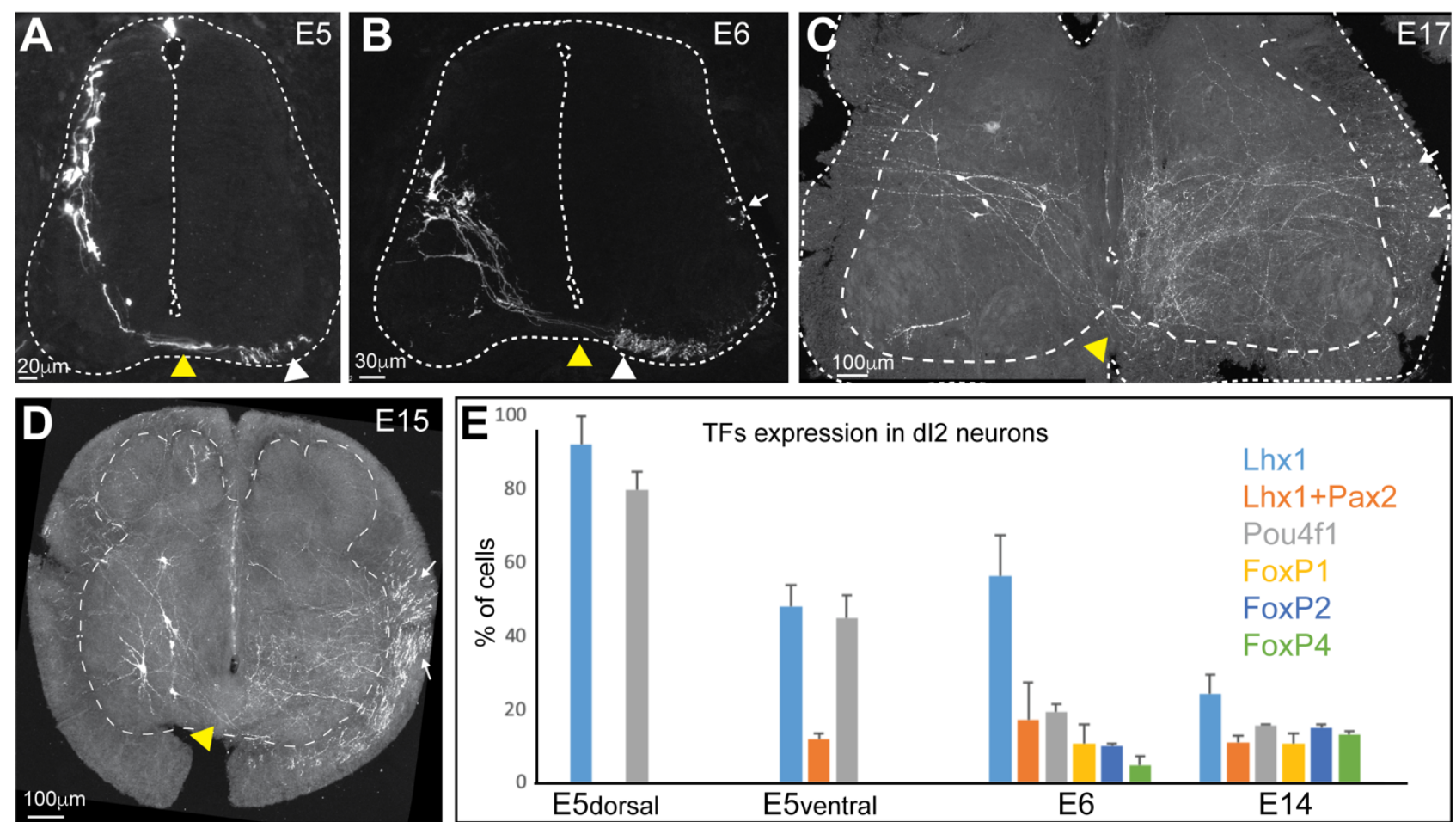

(n)
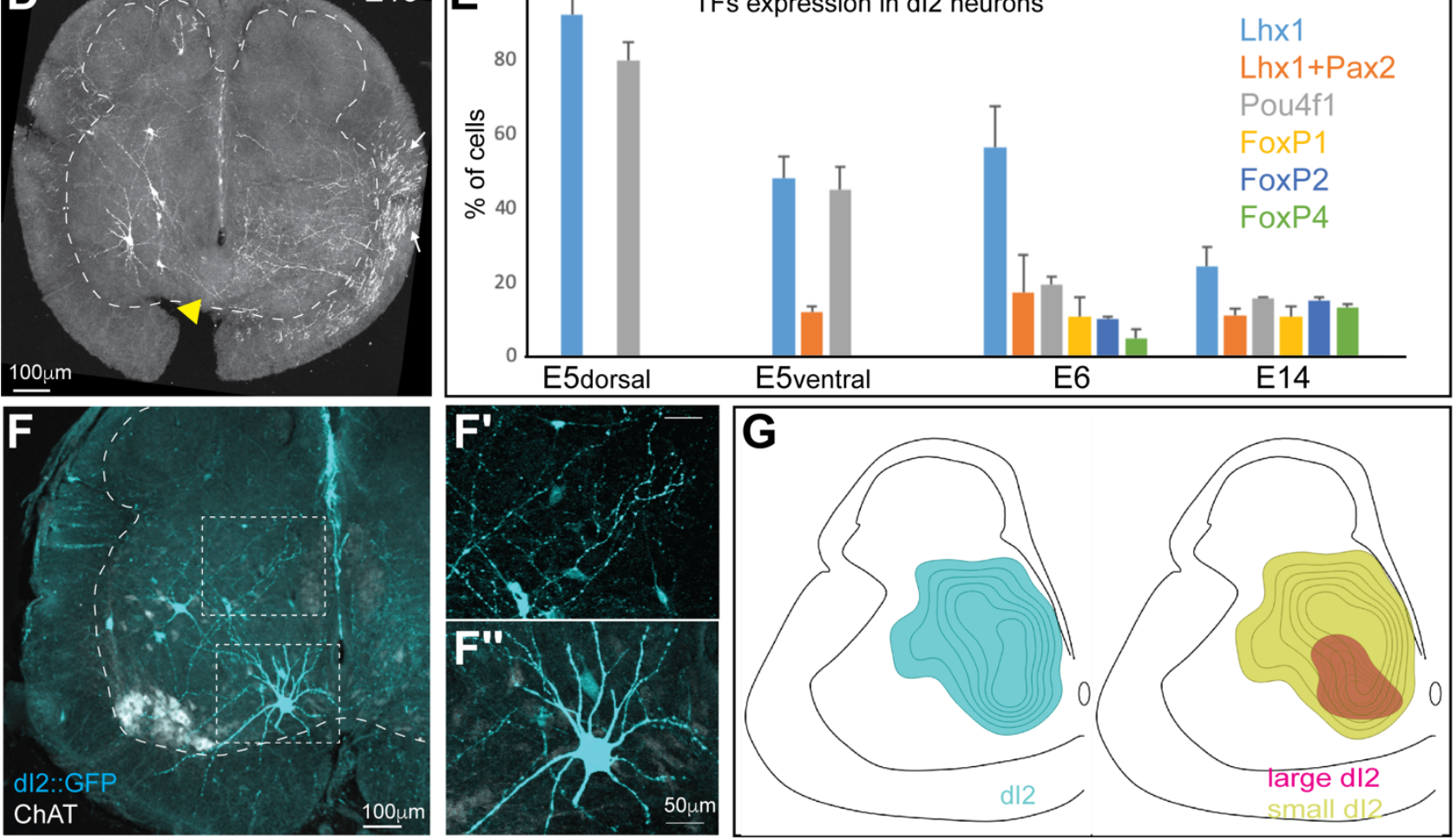

Figure 1: Characterization and classification of dI2 neurons during embryonic development.

dI2 interneurons were labeled by genetic intersection between Foxd3 and Ngn1 enhancers (Avraham et al., 2009)

(Supp Fig. S1).

A-D. dI2 axonal projection during development. At E5 (A) post mitotic dI2 neurons assume a dorsolateral position and start to migrate ventrally. At E6 (B) dl2 neurons occupy the mid-lateral domain. At E15-17 dl2 neurons are located at the medial lamina VII at the lumbar level (LS3) (C) and the thoracic level (T1) (D). dI2 axons cross the floor plate (yellow arrowheads), turn longitudinally at the ventral funiculus (white arrowheads) and eventually elongate at the lateral funiculus (white arrows).

E. A graph indicating the fraction of $\mathrm{d} 12$ neurons expressing TFs during development (based on data from three E5, two E6 and two E14 embryos). F. Cross section of an E17 embryo at the lumbar spinal cord (crural plexus level, LS2). Small-diameter dI2 neurons residing in lamina VII ( $F^{\prime}$ ) and ventromedial large-diameter dI2 neurons in lamina VIII (F"). G. Density plots of dI2 somata in the sciatic plexus level (cyan, $N=374$ cells), dI2 large (magenta) and $\mathrm{d} \mid 2_{\text {small }}$ (yellow) INs ( $N=33$ and $N=344$ cells, respectively, from 2 embryos).

See Figure $\mathrm{S} 2$ and $\mathrm{S} 3$. 
percentage of Gad2 and Slc6a5 dl2-expressing cells was found also in mouse (Delile et al.,

140 2019). At E13-17 in the caudal lumbar level and at the level of the sciatic plexus, most dI2

141 neurons reside at the medial aspect of lamina VII. About $91 \%$ of dI2 neurons are small-

142 diameter neurons that reside at the lateral dorsal aspect of lamina VII, and 9\% are large-

143 diameter neurons. At the lumbar sciatic plexus level, large-diameter dI2 neurons mostly

144 reside at the ventral aspect of lamina VII (Fig. $1 F, G)$ and at the level of the crural plexus in

145 the ventral and dorsal aspect of lamina VII (Fig. S3E). Importantly, large-diameter dI2

146 neurons are only apparent at the lumbar level (Fig. 1F, S3E-F). The division of large- and

147 small-diameter lumbar dI2 neurons was not reflected in the expression of the tested TFs.

148 Hence, dI2 neurons consist several sub-populations, as it was shown with other spinal

149 interneurons (Bikoff et al., 2016, Delile et al., 2019, Sweeney et al., 2018)

151 Sub-population of $\mathrm{d} / 2$ neurons project to the cerebellum

152 To study the supraspinal targets of $\mathrm{dl} 2$ neurons, axonal and synaptic reporters were

153 expressed in lumbar dI2 neurons (Fig. 2A). At E3, dI2 enhancers were co-electroporated with

154 double conditional axonal reporter - membrane tethered cherry, and synaptic reporter -

155 SV2-GFP (Fig. S1A). Expression into the lumbar spinal cord was attained by using thin

156 electrodes positioned near the lumbar segments. At E17, the stage in which the internal

157 granule layer is formed in the chick cerebellum, the axons and synapses of dI2 neurons were

158 studied. dI2 axons cross the spinal cord at the floor plate at the segmental level, ascend to

159 the cerebellum, enter through the superior cerebellar peduncle, and cross back to the other

160 side of the cerebellum ipsilaterally to the targeted dI2s (Fig. 2B). Synaptic boutons are

161 noticeable in the granule layer at the ipsilateral and contralateral sides of the anterior 
162

163

164

165

166 belonged to dI2 (Fig. S4H). Thus, the large diameter dI2s are $10 \%$ of the VSCT neurons, consistent with the anatomical observation that VSCT comprise a heterogeneous population

cerebellar lobules (Fig. 2C). Synaptic boutons were also present in the central cerebellar nuclei (Fig. S4A).

The difference in the soma size between the dorsally and ventrally located $\mathrm{dl} 2$ neurons prompted us to test which dI 2 neurons project to the cerebellum. The $d 12$ and precerebellar neurons were co-labeled by genetic targeting of $\mathrm{d} 12$ at early stages of embryogenesis (E3), coupled with intra-cerebellar injection of cholera toxin subunit B (CTB) or replication-defective HSV-LacZ at E15 (Fig. 2A). Spinal neurons retrogradely-labelled from the cerebellum consist of the double crossed VSCT and the ipsilaterally projecting DSCT neurons. However, dI2 neurons, double labelled by genetic targeting and back labelling from the cerebellum, are all VSCT neurons, since dI2 are commissural neurons. Only the largediameter neurons were co-labeled, most of them in the ventral aspect of lamina VII (Fig. 2D, E,H; S4B,C). Interestingly, many of $\mathrm{CTB}^{+}$or $\mathrm{LacZ}^{+}$neurons were contacted by $\mathrm{dl} 2$ axons (Fig. 2F,G,I; S4D,E), suggesting that small-diameter dI2 neurons innervate pre-cerebellar neurons.

Segmental crossing at the lumbar level and re-crossing back to the ipsilateral side at the cerebellum, is a characteristic of the VSCT projection pattern. To measure the proportion of $\mathrm{dI} 2$ in VSCT neurons, we labeled VSCT axons with GFP and dI2 axons with cherry (for experimental design see Supp. Fig. S4G,F). The number of axons expressing the reporters at the contralateral superior cerebellar peduncle was scored. Ten percent of the VSCT axons of interneurons (Jankowska and Hammar, 2013, Stecina et al., 2013). 

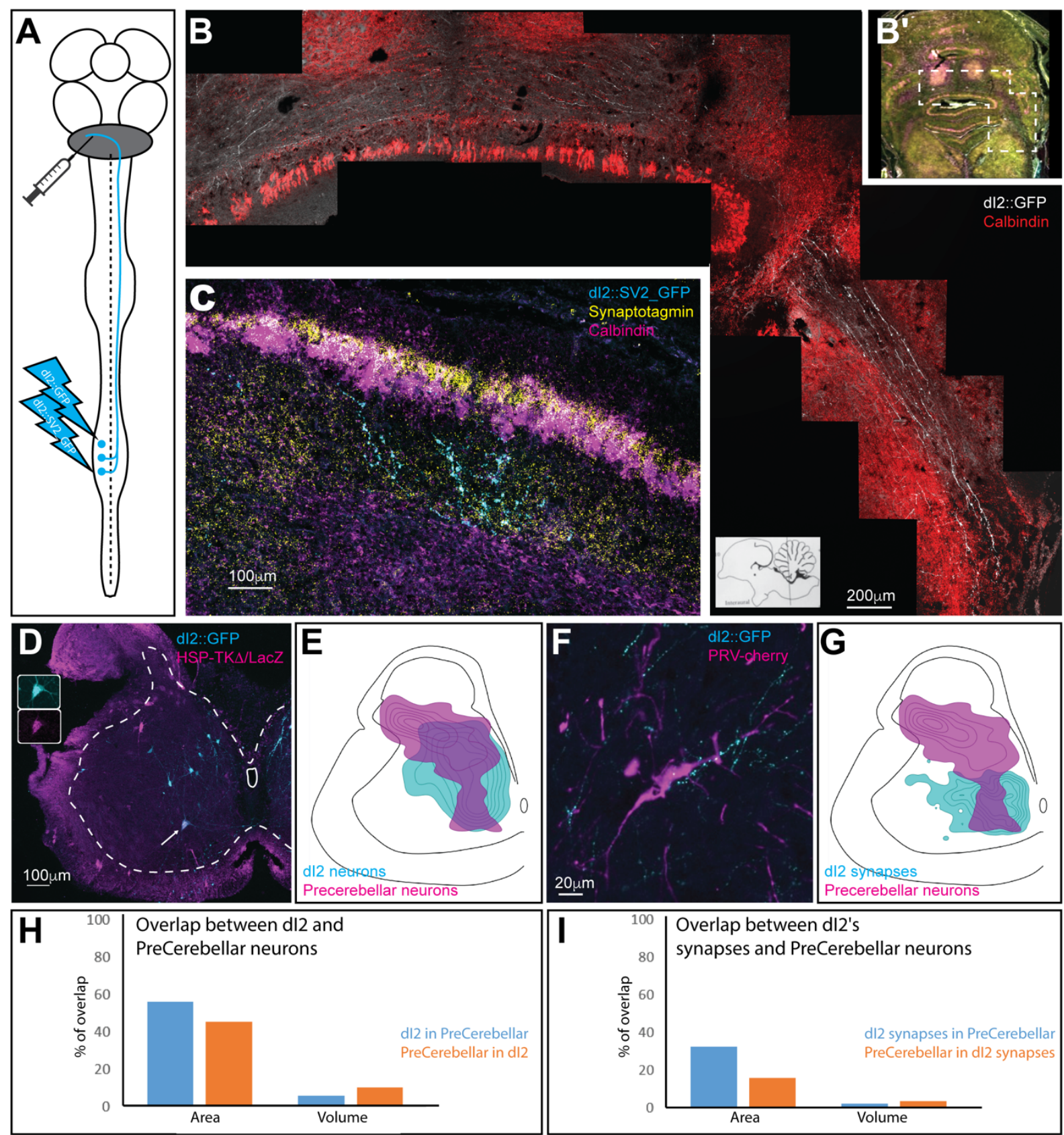

185 Figure 2: $\mathrm{dl} 2$ neurons project to the cerebellum.

A. Experimental setup for labeling of cerebellar projecting dl2 neurons. dl2 neurons were genetically targeted at $\mathrm{HH} 18$, and pre-cerebellar neurons were labeled using intra-cerebellar injection of CTB or replication defective HSV-LacZ at E15. B. A cross section of E17 brainstem and cerebellum. The dashed polygon in B' is magnified in $B$. dl2 axons reach the cerebellum, enter into it via the superior cerebellar peduncle and cross the cerebellum midline. Calbindin (Purkinje neurons, magenta ( $\left.B^{\prime}\right)$ or red (B)), synaptotagmin (yellow). C. A cross section of E17 cerebellar cortex. Lumbar-originating dI2 synapses (cyan) in the granular layer of the anterior cerebellar cortex. Calbindin (Purkinje neurons, magenta), synaptotagmin (yellow). D. A cross section of an E15 embryo at the lumbar spinal cord level (sciatic plexus level). Pre-cerebellar neurons were infected and labeled by HSV-LacZ (magenta), and dI2 neurons express GFP (cyan). A large-diameter d12 neuron co-expressing LacZ and GFP is indicated by an arrow (magnification of the two channels in the insets). E. Density plots of dI2 and pre-cerebellar neurons (density values 10-90\%) in the sciatic plexus segments ( $N=374$ and $N=289$ cells, respectively). F. CTB labeled pre-cerebellar neuron (magenta) is contacted by dI2 axonal terminals (cyan). G. Density plots of dI2 synapses and pre-cerebellar neuron somata (density values 10-90\%) in the sciatic plexus segments ( $N=4735$ synapses and $N=289$ cells, respectively). $H, I$. Quantification of the overlap in area and volume of the two density plots. The plots are based on data from 3 embryos. See Figure S4. 
187 To assess the synaptic input to dI2 neurons we investigated their synaptic connectivity with

188 known pre-VSCT neurons: dorsal root ganglion (DRG) neurons, inhibitory and excitatory pre-

189 motoneurons and reticulospinal tract neurons. Two genetically defined pre-motoneurons

190 were examined: V1 - an inhibitory pre-motoneuronal population (Bikoff et al., 2016,

191 Gosgnach et al., 2006) and dI1i excitatory INs. A density plot of dI1 synapses shows dI1i

192 terminals within the motoneuron pools (lamina IX) and at lamina VII (Fig. 3C). Co-labeling of

193 motoneurons and dI1 synapses revealed synaptic contacts of dI1i on motoneurons (Fig. S5A-

194 B). This was further supported by co-labeling dI1 with pre-MNs via hindlimb injection of

195 pseudorabies virus (PRV) (Fig. S5C-D).

To identify neurons presynaptic to dI2 cells we initially assessed the likelihood of

197 connectivity by examining spatial overlap of axonal terminals from the presumed

198 presynaptic neurons and the somata of $\mathrm{dI} 2$ neurons, and consequently by detection of

199 synaptic boutons on the somatodendritic membrane of dI2 neurons. We have shown

200 previously that genetic targeting of synaptic reporter in chick spinal cord neurons, yields

201 overlapping expression of the reporter and endogenous pre-synaptic proteins (Hadas et al.,

202 2014). This observation was confirmed using synaptic reporter expressed in dI1 and

203 cytoplasmic reporter expressed in dI2 (Fig. 3C'). The dI2, DRG, V1, and dI1 neurons were

204 labeled using specific enhancers (Fig. S1A). General premotor INs were labeled by injection

205 of PRV-cherry virus into the ipsilateral hindlimb musculature (Hadas et al., 2014) (Fig. 3B).

A density profile of the axons of DRG neurons (Fig. 3A, S6A-D) and PRV-labeled pre-

207 MNs (Fig. 3B), and a density profile of synaptic boutons from dl1i (Fig. 3C, S6E), V1 (Fig. 3D),

208 and reticulospinal tract neurons $\left(5 \mathrm{HT}^{+}\right.$synapses) (Fig. 3E, $\left.\mathrm{S} 6 \mathrm{~F}\right)$ were aligned to the density

209 plots of the dl2 somata. The overlap between the axonal terminals of DRG neurons, PRV- 
210 labeled pre-motoneurons, dI1 i and V1 boutons, and the somata of dI2 neurons is evident

211 (Fig. 3A-F, Fig. S6G). To further substantiate these putative synaptic connections, we

212 performed double labeling of DRG, PRV-labeled pre-motoneurons, V1, and dI1i, together

213 with dI2 neurons. Hindlimb pre-motor neurons were labeled by injection of PRV into the

214 hindlimb musculature (Hadas et al., 2014); DRG and dI1 terminal and synapses by cell-type

215 specific enhancer (Fig. S1A); V1 synapses by a double conditional on/off reporter plasmids

216 that enables activation of synaptic reporter in V1 and dI2 utilizing the Foxd3 enhancer and

217 deletion of the synaptic reporter from dI2 neurons utilizing the Ngn1 enhancer (Fig. S1A).

218 Contact between DRG axons and dI2 neurons was mainly apparent in the dorsal dI2

219 neurons, while the ventral dI2 neurons received little to no input from DRG neurons (Fig. 3A,

220 S6A,B). Synaptic connections, evaluated by boutons on dI2 dendrites and somata, are

221 apparent from the pre-motor neurons V1 and dI1i (Fig. 3C,D). Serotonergic synapses were

222 found to be concentrated on motoneurons and were not observed on dI2 neurons (Fig. 3E,

223 S6F). Double-labeling of 5HT and dI2 neurons did not reveal any synaptic input. The lack of

224 synaptic serotonergic input may be related to the difference in species, or may suggest that

225 other, non-dI2 VSCT neurons residing adjacent to motoneurons, are contacted by the

226 reticulospinal neurons. The analysis of the synaptic inputs supports the concept that dI2

227 neurons constitute part of the VSCT. They receive input from sensory afferents, inhibitory

228 and excitatory pre-motoneurons and project to the cerebellum. 

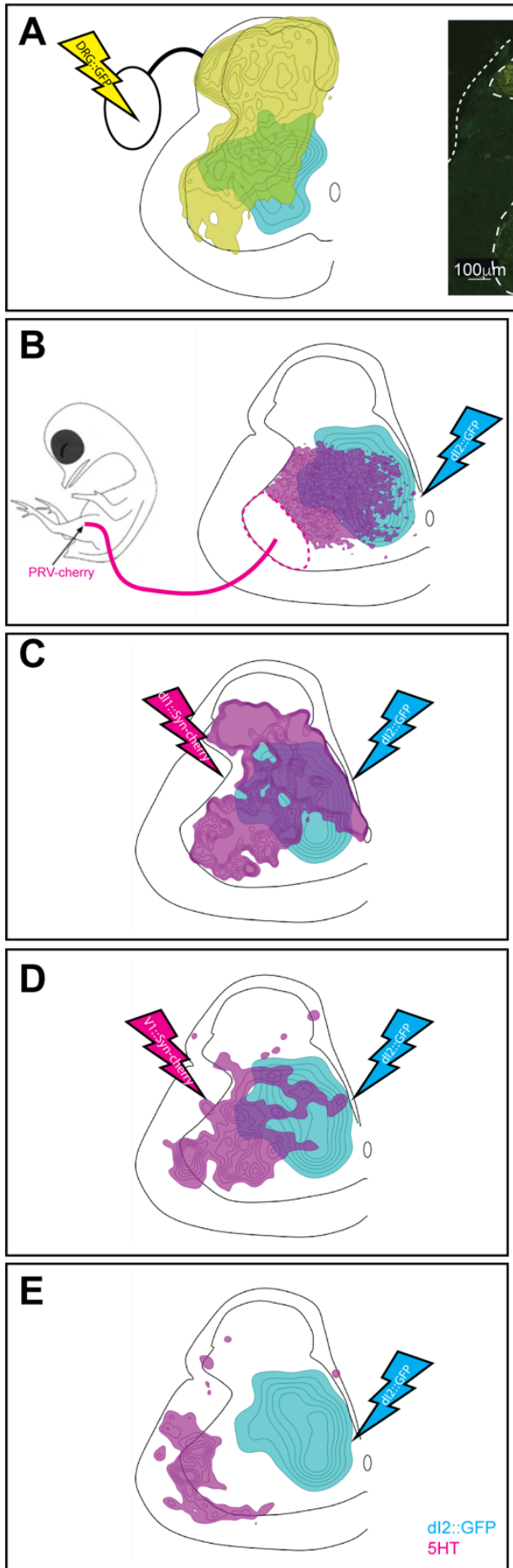
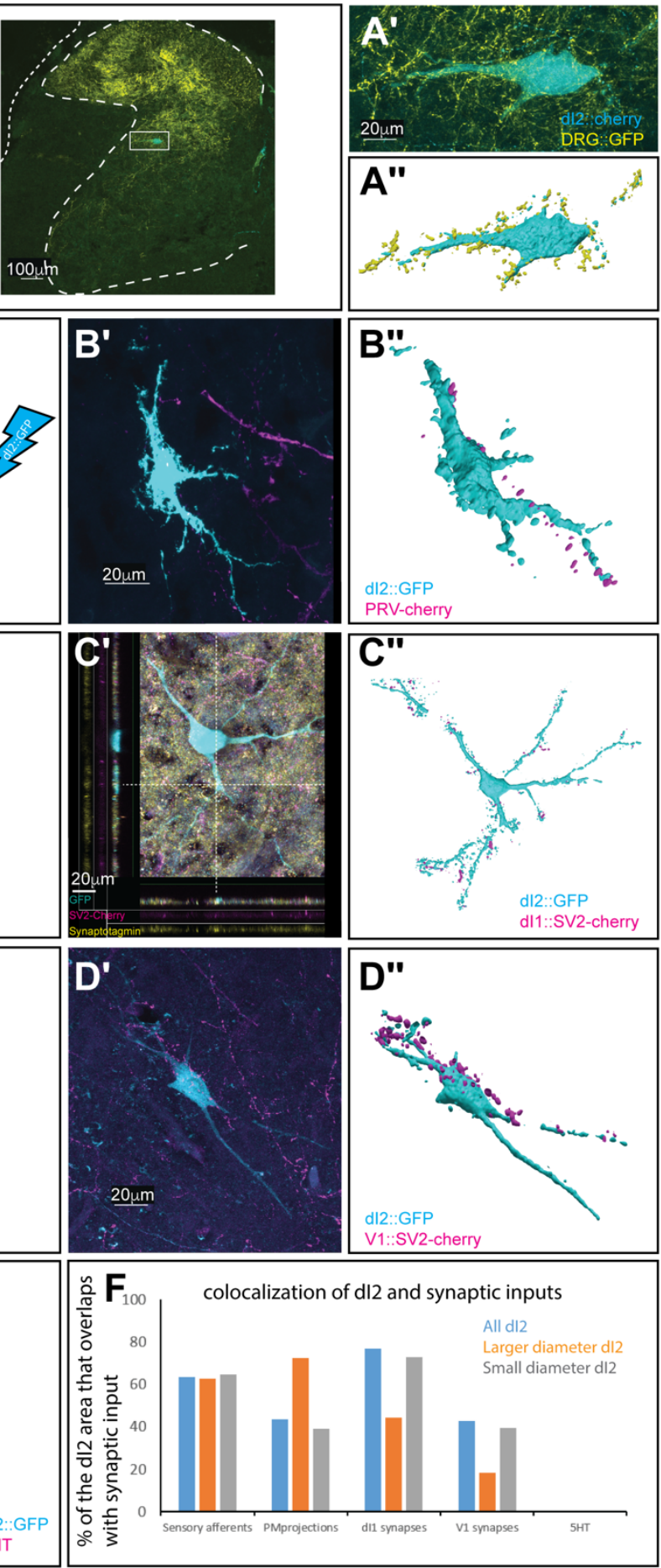

Figure 3: Synaptic inputs to d12 neurons.

Schematic representations of the experimental design for labeling dI2::GFP or dI2::cherry INs (cyan) and potential sources of synaptic inputs (yellow or magenta), supplemented by cell soma density of dI2 INs and the synaptic densities are illustrated in A, B, C, D, E. The density values presented are $10-80 \%, 20-80 \%, 25-80 \%, 30-50 \%$ and $20-80 \%$, respectively.

Examples of d12 neurons contacted by axons or synaptic boutons are shown in $A^{\prime}, B^{\prime}, C^{\prime}$, and $D^{\prime}$; and their 3D reconstruction in $A^{\prime \prime}, B^{\prime \prime}$, $C^{\prime \prime}$, and $D^{\prime \prime}$. Genetic labeling was attained using specific enhancers (Fig. S1) electroporated at $\mathrm{HH} 18$.

A. DRG neurons form contacts on dI2 neurons. Inset in A: cross section of and E17 embryo at the crural plexus level of the lumbar cord. A dorsally located dI2 neuron contacted by numerous sensory afferents, magnified in $A^{\prime}$ and 3D-reconstructed in $A^{\prime \prime}$. ( $N=18$ sections)

B. Premotoneurons form contacts on $\mathrm{dl} 2$ neurons. dI2 neurons were labeled at HH18. At E13, PRV virus was injected to the leg musculature, and the embryo was incubated until premotoneuronal infection ( 39 hours) ( $N=34$ sections).

C. dl1 neurons form synapses on dI2 neurons. ( $N=8568$ synapses). $C^{\prime}$ : A representative SV2::cherry synapse on dI2 dendrite, positive for synaptotagmin. Demonstrated by a horizontal and vertical optical sections in Z-axis (see cursors and color channels).

D. V1 neurons form synapses on dI2 neurons. ( $N=1923$ synapses).

E. $\mathrm{dl} 2$ neurons do not have $5 \mathrm{HT}$ synaptic terminals ( $N=1718$ synapses). E17 cross sections of dI2::GFP labeled embryos were stained for $5 \mathrm{HT}$.

F. Quantification of the overlap area of the different input sources and dI2 neuron densities plots. See Figure S5, S6. 
232 Axon collaterals of $\mathrm{d} I 2$ invade the spinal cord gray matter along the entire length of the

233 spinal cord, as revealed by whole mount staining of spinal cords electroporated with alkaline

234 phosphatase reporter (dI2::AP) (Fig. 4A) and membrane-tethered EGFP (Fig. 4B). The region

235 innervated by dI2 collaterals (arrow at Fig. 4B) overlaps with that of the pre-MNs, V0 and V1

236 (Lai et al., 2016, Griener et al., 2015), as well as with that of the contralateral dI2 neurons

237 (Fig. 1, Fig. 4B). To assess the potential spinal targets of dl2 neurons, we scored the degree

238 of overlap between of $\mathrm{dl} 2$ synapses and dI2 somata (Fig. 4C), the ipsilateral pre-MNs (Fig.

239 4D), and contralateral pre-MNs (Fig. 4E). The alignment revealed a significant overlap of dI2

240 synapses with ipsi/contralateral pre-MNs and dI2 neurons (Fig. 4G,H). Co-labeling of synaptic

241 dI2 coupled with labeling of the above neuronal population showed dI2 synaptic boutons on

242 pre-MNs and dI2 neurons at the lumbar level (Fig. 4C-H, S7A-C).

243 The pattern of dI2 collaterals along the entire rostrocaudal axis (Fig. 4A) suggests that

244 dI2 neurons innervate contralateral pre-MNs and dI2 neurons at multiple levels. To test this,

245 labeling of lumbar dI2 neurons was coupled with labeling of brachial pre-MNs and dI2 soma

246 labeling via wing musculature injection of PRV or electroporation of reporter in brachial dI2

247 neurons, respectively (Fig. 4F, S7D). dI2-synapses and the putative targets overlapped, and

248 synaptic boutons originated from lumbar level dI2 neurons are apparent on dI2 neurons, and

249 on the contra- and ipsilateral pre-MNs of the wings (Fig. 4F, S7D-F).

The neuronal and synaptic labelling experiments showed that lumbar $\mathrm{d} / 2$ neurons

251 innervate the cerebellum, lumbar and brachial pre-MN, and contralateral dI2 neurons (Fig.

252 6D). Hence, dI2 neurons may relay peripheral and intraspinal information to the cerebellum

253 and to the contralateral lumbar and brachial motor control centers. 

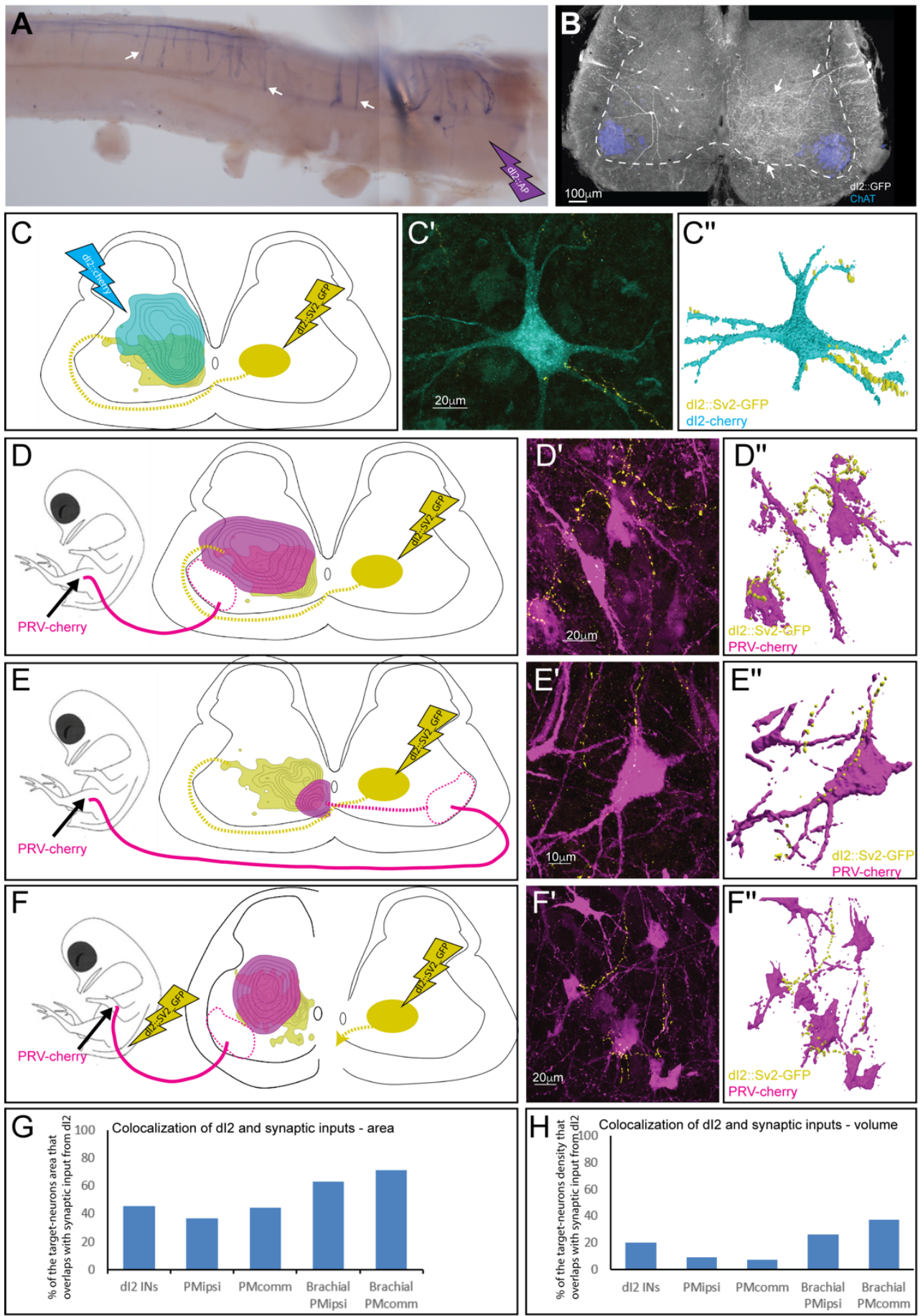

254

Figure 4: Spinal synaptic targets of dI2 neurons.

A. A whole mount staining of spinal cord (thoracic segments) expressing alkaline phosphatase (AP) in dl2 neurons. The lumbar dl2 neurons (not included in the image) were labeled with AP. dI2 axon collaterals project and into the spinal cord (arrows).

B. Cross section of an E17 embryo at the crural plexus level of the lumbar cord. The axon collaterals (white arrow) penetrating the gray matter of the contralateral side are evident.

Schematic representations of the experimental design for labeling synapses (dI2::SV2-GFP, yellow) and potential targets (magenta) supplemented by cell soma density and $\mathrm{dl} 2$ synaptic densities are illustrated in $\mathrm{C}, \mathrm{D}, \mathrm{E}$, and $\mathrm{F}$. Examples of target neurons contacting synaptic boutons of dl2 neurons are shown in $C^{\prime}, D^{\prime}, E^{\prime}$, and $F^{\prime}$; and their 3D reconstruction in $C^{\prime \prime}, D^{\prime \prime}, E^{\prime \prime}$, and $F^{\prime \prime}$. Genetic labeling was attained using dI2 enhancers (Fig. S1) electroporated at HH18. Pre-MNs were labeled by injection of PRV-cherry into the hindlimbs (D, E) or the forelimb (F) musculature, at E13. The embryos were incubated until pre-MNs infection (39 hours).

C. dI2 neurons innervate the contralateral dI 2 neurons ( $N=4735$ synapses and $N=374$ cells, respectively).

D. $\mathrm{dl} 2$ neurons innervate ipsilateral projecting premotoneurons at the sciatic plexus level ( $N=4735$ synapses and $N=936$ cells, respectively).

E. dI2 neurons innervate contralateral projecting premotoneurons at the sciatic plexus level ( $N=4735$ synapses and $N=47$ cells, respectively).

F. dl2 neurons innervate brachial ipsilateral projecting premotoneurons ( $N=2215$ synapses and $N=286$ cells, respectively).

G. Quantification of the overlap area of different synaptic targets and dI2 synapse density plots, as the percentage of overlap between dI2 synapses and the target.

H. Quantification of the overlap in volume of the different synaptic targets and dI2 synapse density plots, as the percentage of overlap between the synaptic target and dI2 synapses. See Figure S7. 
The synaptic input to $\mathrm{d} I 2$ and their targets, implicate them as relaying information about motor activity to the contralateral spinal cord and the cerebellum. Thus, we hypothesized that manipulation of their neuronal activity may affect the dynamic profile of stepping.

To study the physiological role of dI2 neurons, we silenced their activity using bilateral targeting of the tetanus toxin (TeTX) gene, to lumbar dI2s. EGFP was co-targeted in a $2 / 1$ TeTX/EGFP ratio. EGFP expression in dI2 neurons and non-electroporated chicks was used as a control. In order to maximize the number of targeted dI2 neurons, we combined genetic lumbar spinal cord (Fig. S1A). Embryos were electroporated at E3. Upon hatching, chicks were trained for targeted over-ground locomotion. electroporation (Supplementary table S1). balance on a tilted meshed surface. TeTX-manipulated chicks and control chicks, maintained

277 balance on the titled surface up to $63-70^{\circ}$, with no apparent statistically significant 278 differences (Supplementary Table S1, Supplementary statistics). Thus, manipulation of dI2 279 neuronal activity did not impair post-hatching development and muscle strength. 

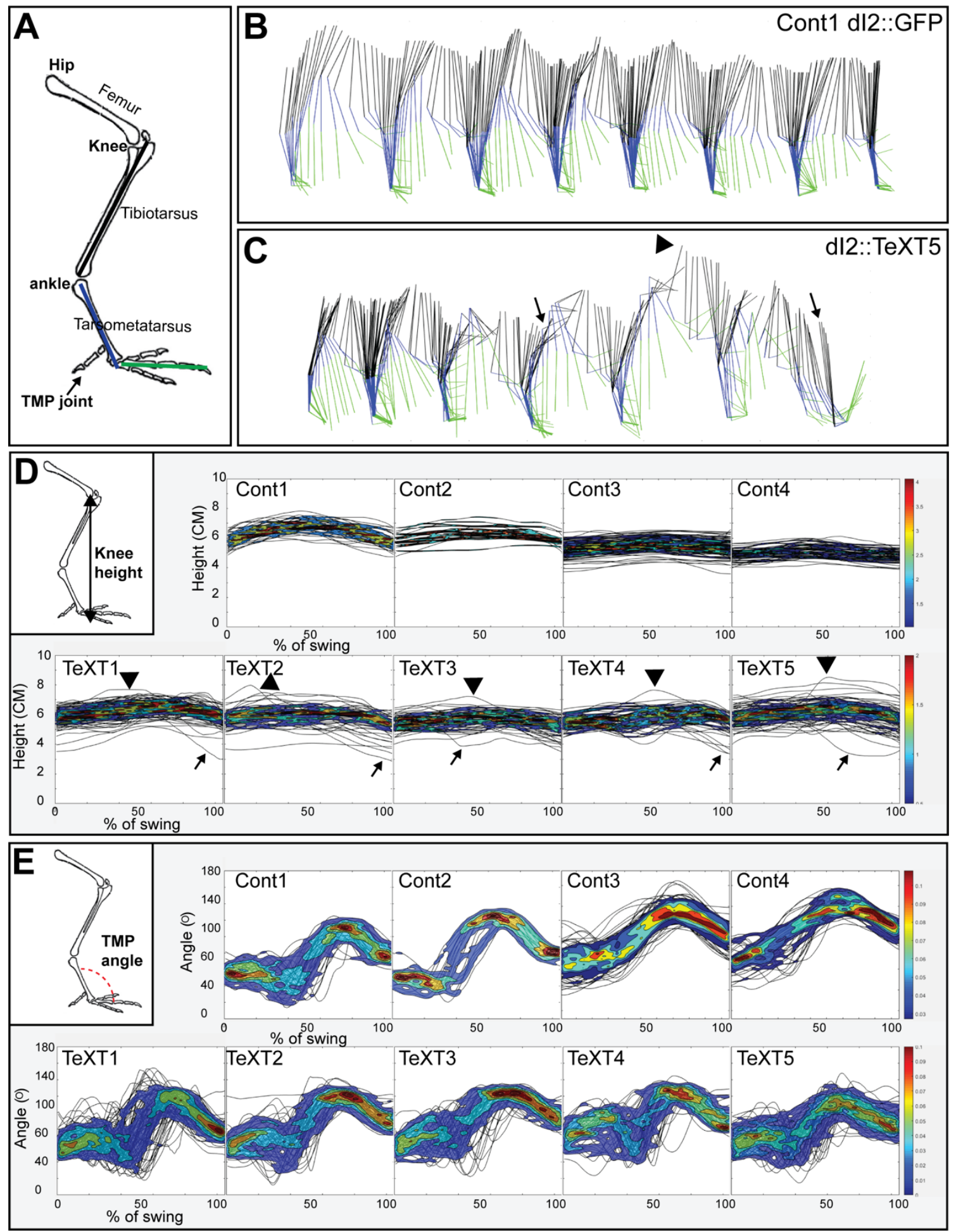

Figure 5: Kinematic analysis of locomotion in post-hatching chicks following neuronal silencing of dI 2.

A. Schematic illustration of chick hindlimb joints (bold) and bones (regular). The knee joint connects between the femur and the tibiotarsus, the ankle connects the tibiotarsus and the tarsometatarsus which connects to the phalanges by the tarsometatarso-phalangeal joint (TMP). During the swing phase of birds, the ankle flexion leads to foot elevation, while the knee is relatively stable.

B,C. Stick diagrams of stepping in a control chicken d2::GFP (B) and in a d2::TeTX chicken (C). Arrows indicate falls and overshoots are denoted by arrowheads.

D. Overlays of knee height (demonstrated in insert) trajectories during the swing phase in all analyzed steps of each of the control and TeTX treated P8 hatchlings are shown superimposed with the respective $20 \%-80 \%$ color coded density plots as a function of the percentage of swing (see Text and Materials and Methods). Arrows indicate falls and overshoots are indicated by arrowheads.

E. Overlay of angular trajectories of the TMP joint (demonstrated in insert) during the swing phase in all analyzed strides of each of the control and TeTX treated P8 hatchlings are shown superimposed with the respective $20 \%-80 \%$ color coded density plots as a function of the percentage of swing (see Text and Materials and Methods). 
was found during stepping in all the manipulated and the control chicks (Fig. S8A, table 1,

,tables 3, Supplementary statistics).

289 Whole-body collapses: A collapse was scored as a decline of the knee height below $85 \%$ of

290 the average knee height at the stance phase of the step (arrow in Fig. 5C). Collapses were

291 usually followed by over-extensions (arrow head in Fig. 5C). We measured the number of

292 collapses in 50-190 steps. In control chicks, collapses occurred in $0.53 \pm 0.92 \%$ of the steps. In

293 TeTX manipulated chicks we observed collapses in $19.46 \pm 8.3 \%$ of the steps, significantly

294 different from the controls (Fig. 6A, Supplementary statistics). The collapses and over-

295 extensions were also manifested in the profiles of the knee height trajectory during the 296 swing phase (Fig. 5D).

297 Wide base stepping: Wide-base stance is typical of an unbalanced ataxic gait. The stride 298 width was measured between the two feet during the double stance phase of stepping. The 299 mean stride in TeTX-manipulated chicks 1,2,4, and 5 was significantly wider than the control 300 chicks, while the width in TeXT3 was similar to the controls (Table 2, Supplementary 301 statistics).

302 Variable limb movements: In stable gait, limb trajectories are consistent from stride to 303 stride. For point by point comparisons of the trajectories in knee height and angles of the 304 TMP joint during the swing phase of stepping in control and TeTx manipulated chicks, the 
swing phase was normalized using 126 consecutive epochs, and the data were displayed as a

306 function of the percentage of swing. Plots of the knee height and TMP angle trajectories

307 during the normalized swing in all the analyzed steps of each chick are shown superimposed

308 in Fig. 5D and Fig. 5E, respectively. These data demonstrate that the range of changes in

$309 T e T X$-manipulated chicks was higher comparing to control chicks.

311 and TMP angle ranges than the TeXT-treated group, even though there are differences

312 within groups (Fig. S8B,C). The average knee height range of the combined control chicks

$313(1.981 \pm 0.33)$ is significantly lower than the range of the combined TeXT treated chicks

314 (3.109 \pm 0.74$)$ (Fig. 6B, Supplementary statistics). Similar comparison of the combined ranges

315 of angular excursions of the TMP joint during the normalized swing revealed that the

316 average angle of the controls group (49.34 \pm 16.03$)$ is significantly lower than the average of

317 the TeXT-treated chicks (77 \pm 22.149$)$. (Fig. 6C, Supplementary statistics).

318 Since the increased range of changes could be due to the effects of the substantial

319 increase in body collapses during stepping (Fig. 6A, see also Fig. 5), we excluded steps

320 featuring whole body collapses and reanalyzed the data. The data summarized in table 3,

321 shows that the significant difference between controls and the TeXT-treated chicks in the

322 range of the knee height and the TMP angle excursions was maintained. Thus, the increase

323 in irregularity in the TeXT-treated chicks is not only due to the body collapses of the TeXT-

324 treated chicks. Overall, the kinematic parameters of the dI2-TeXT-treated chicks

325 demonstrate a reduction in stability during locomotion, featuring a possible role of dI2 in

326 stabilizing bipedal stepping. 


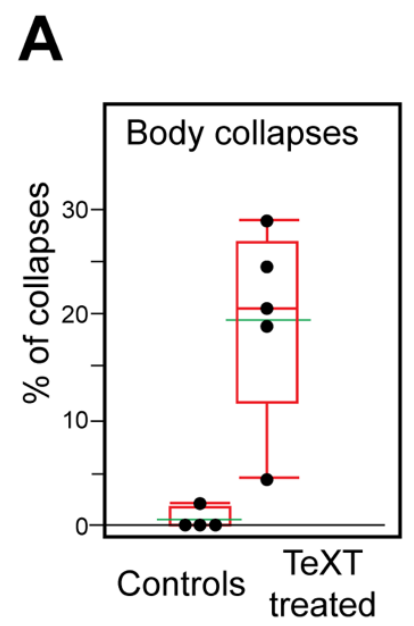

\section{B}

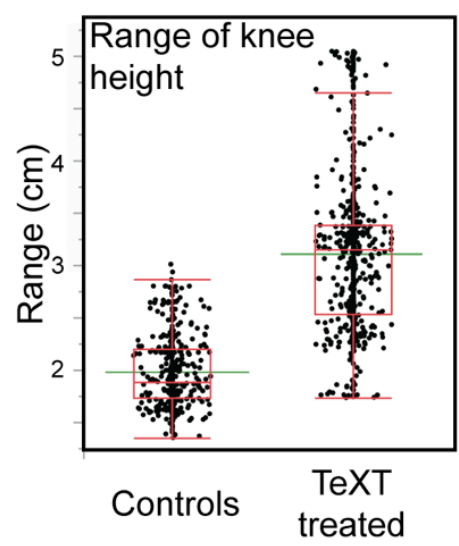

C

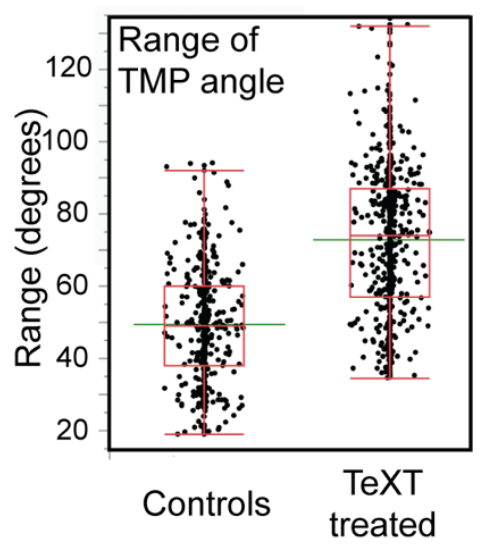

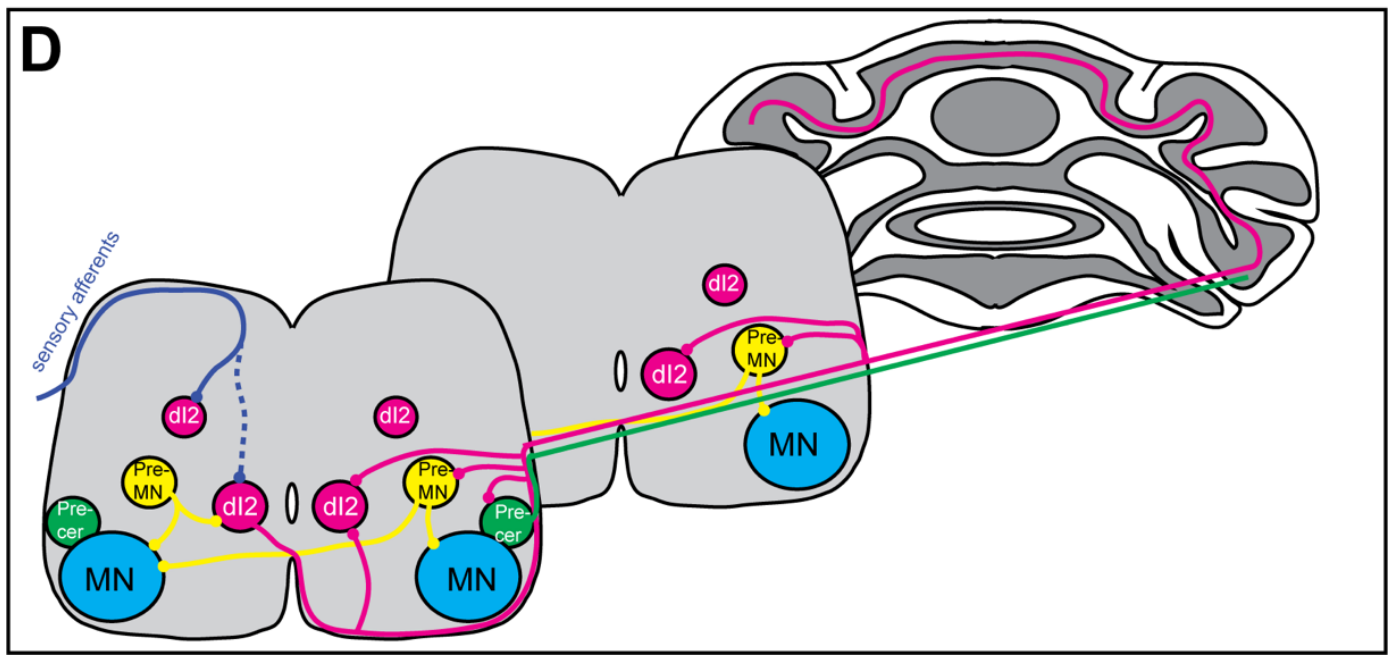

328 Figure 6. Increased inter-stride variability in TMP angle and knee height in TeTX-treated chicks.

A. The percentage of steps with body collapses in the controls and TeTX manipulated hatchlings ( $n=4$ and $n=5$, respectively). $p$-value $<0.0001$ (Z-test). See table 3 for single chick fall proportions.

B. Analysis of the mean range of knee height changes during the swing phase of control and TeTX treated chicks ( $n=4$ and $n=5$, respectively). $p$-value $<0.0001$ using a T-test allowing different variances.

See Figure S8B and table 3 for single chick data and statistical analysis details.

C. Analysis of the mean range of TMP angular excursions during the swing phase of control- and TeTXtreated chicks. ( $n=4$ and $n=5$, respectively). $p$-value $<0.0001$ Watson and Williams $F$ test.

See Figure $\mathrm{S} 8 \mathrm{C}$ and table 3 for single chick data and statistical analysis details.

D. Schematic illustration showing the connectome of lumbar dI2 neurons. dl2s (magenta) receive synaptic input from sensory afferents (solid blue line indicates massive synaptic input and dashed blue line indicates sparse innervation), Inhibitory and excitatory premotoneurons (yellow), and from the contralateral lumbar dI2. dI2s innervate the contralateral lumbar and brachial premotoneurons (both commissural and ipsilateral projecting premotoneurons are innervated by dI2), the lumbar and brachial contralateral dl2, lumbar pre-cerebellar neurons (green) and the granule neurons in the cerebellum. 
329 Table 1: Stride velocity and left-right phase in control and TeTx manipulated chicks

330 Swing velocity and left-right phase were measured and calculated as described in the

331 Materials and Methods. The Watson and Williams F test of the phase data (circular ANOVA)

332 were not statistically significant (See also Supplementary statistics).

333

\begin{tabular}{|c|c|c|c|}
\hline chick & $\begin{array}{c}\text { Ave. Swing } \\
\text { velocity }(\mathrm{cm} / \mathrm{sec})\end{array}$ & Mean left-right phase $\left(^{\circ}\right)$ & \# of steps \\
\hline TeTX1 & $46.78 \pm 22.13$ & $184.679 \pm 33.003$ & 113 \\
\hline TeTX2 & $62.24 \pm 20.17$ & $182.293 \pm 32.01$ & 63 \\
\hline TeTX3 & $48.06 \pm 20.04$ & $180.784 \pm 31.064$ & 69 \\
\hline TeTX4 & $57.24 \pm 24.35$ & $180.502 \pm 36.291$ & 59 \\
\hline TeTX5 & $36.66 \pm 17.61$ & $181.97 \pm 35.787$ & 93 \\
\hline Control 1 (GFP) & $79.65 \pm 37.77$ & $182.369 \pm 35.366$ & 47 \\
\hline Control 2 (GFP) & $41.91 \pm 20.41$ & $182.384 \pm 26.708$ & 19 \\
\hline $\begin{array}{c}\text { Control3 (Not } \\
\text { electroporated) }\end{array}$ & $41.09 \pm 16.59$ & N.D. & 121 \\
\hline $\begin{array}{c}\text { Control4 (Not } \\
\text { electroporated) }\end{array}$ & $42.3 \pm 30.91$ & N.D. & 51 \\
\hline
\end{tabular}

334 
335 Table 2: Maximum stride width in control and TeTx manipulated chicks

336 Stride width was measured as described in Materials and Methods. One-way ANOVA

337 followed by Dunnett's.

338

\begin{tabular}{|c|c|c|}
\hline chick & maximum stride width $(\mathrm{cm})$ & $\begin{array}{c}\text { \# of } \\
\text { steps }\end{array}$ \\
\hline TeTX1 & $5.11 \pm 1.89$ & 97 \\
\hline TeTX2 & $5.32 \pm 1.38$ & 36 \\
\hline TeTX3 & $4.5 \pm 1.01$ & 27 \\
\hline TeTX4 & $4.9 \pm 1.16$ & 49 \\
\hline TeTX5 & $5.82 \pm 1.71$ & 110 \\
\hline Control 8 & $4.15 \pm 1.07$ & 137 \\
\hline Control 9 & $4.32 \pm 1.32$ & 115 \\
\hline
\end{tabular}

339 
340 Table 3: Collapses, knee height and TMP angle ranges in control and TeTx manipulated

341 chicks.

342 Analysis of the range between the highest and lowest point of knee height and the highest

343 and lowest angle of the TMP joint in all steps before and after subtraction of collapsed steps.

344 When combined - the difference is statically significant ( $p$-value $<0.0001$ for both metrics,

345 using a T-test allowing different variances).

346

\begin{tabular}{|c|c|c|c|c|c|c|c|c|c|c|}
\hline \multirow[t]{2}{*}{ chick } & \multirow{2}{*}{$\begin{array}{c}\text { \% of } \\
\text { steps } \\
\text { with } \\
\text { collaps } \\
\text { e }\end{array}$} & \multicolumn{4}{|c|}{$\begin{array}{l}\text { TMP angle. } \\
\text { Mean range }\left({ }^{\circ}\right)\end{array}$} & \multicolumn{4}{|c|}{$\#$ of steps } & \multirow{2}{*}{$\begin{array}{c}\% \text { of } \\
\text { steps } \\
\text { with } \\
\text { collaps } \\
\text { e } \\
\end{array}$} \\
\hline & & $\begin{array}{l}\text { All } \\
\text { steps } \\
\text { Ave }\end{array}$ & $\begin{array}{l}\text { Combine } \\
\text { d Ave }\end{array}$ & $\begin{array}{l}\text { Minus } \\
\text { collapses } \\
\text { Ave }\end{array}$ & $\begin{array}{c}\text { Minus } \\
\text { collapses } \\
\text { combine } \\
\text { d Ave } \\
\end{array}$ & $\begin{array}{l}\text { All } \\
\text { steps } \\
\text { Ave }\end{array}$ & $\begin{array}{l}\text { Combined } \\
\text { Ave }\end{array}$ & $\begin{array}{l}\text { Minus } \\
\text { collapses }\end{array}$ & $\begin{array}{c}\text { Minus } \\
\text { collapses } \\
\text { combined } \\
\text { Ave }\end{array}$ & \\
\hline ТeTX1 & 4.4 & $\begin{array}{c}3.05 \\
\pm \\
0.49\end{array}$ & \multirow{5}{*}{$\begin{array}{c}3.11 \pm 0.7 \\
4\end{array}$} & $2.87 \pm 0.38$ & \multirow{5}{*}{$\begin{array}{c}2.83 \pm 0.5 \\
7\end{array}$} & $\begin{array}{c}82.27 \\
\pm 22\end{array}$ & \multirow{5}{*}{$\begin{array}{c}72.71 \pm 20.5 \\
2\end{array}$} & $79.4 \pm 24.5$ & \multirow{5}{*}{$\begin{array}{c}68.97 \pm 21.4 \\
7\end{array}$} & 113 \\
\hline ТeTX2 & 20.60 & $\begin{array}{c}3.5 \pm \\
0.3 \\
\end{array}$ & & $3.35 \pm 0.47$ & & $\begin{array}{c}71.79 \\
\pm 25\end{array}$ & & $71.1 \pm 25.68$ & & 63 \\
\hline ТеTX3 & 18.8 & $\begin{array}{c}2.57 \\
\pm \\
0.31 \\
\end{array}$ & & $2.27 \pm 0.26$ & & $\begin{array}{c}64.17 \\
\pm 21\end{array}$ & & $62.79 \pm 20.9$ & & 69 \\
\hline TeTX4 & 20.45 & $\begin{array}{c}2.54 \\
\pm \\
0.55\end{array}$ & & $\begin{array}{c}2.477 \pm 0.5 \\
6\end{array}$ & & $\begin{array}{c}72.48 \\
\pm 17\end{array}$ & & $66.22 \pm 19.1$ & & 59 \\
\hline TeTX5 & 29 & $\begin{array}{c}3.86 \\
\pm \\
0.83\end{array}$ & & $3.2 \pm 0.2$ & & $\begin{array}{c}72.86 \\
\pm 12\end{array}$ & & $\begin{array}{c}65.85 \pm 13.1 \\
3\end{array}$ & & 93 \\
\hline $\begin{array}{c}\text { Control } 1 \\
\text { (GFP) }\end{array}$ & 2.12 & $\begin{array}{c}1.91 \\
\pm \\
0.22\end{array}$ & \multirow{4}{*}{$\begin{array}{c}1.98 \pm 0.3 \\
3\end{array}$} & $1.91 \pm 0.22$ & \multirow{4}{*}{$\begin{array}{c}1.98 \pm 0.3 \\
3\end{array}$} & $\begin{array}{c}56.8 \pm \\
16.4\end{array}$ & \multirow{4}{*}{$\begin{array}{c}49.34 \pm 16.0 \\
3\end{array}$} & $56.95 \pm 16.6$ & \multirow{4}{*}{$49.4 \pm 16.18$} & 47 \\
\hline $\begin{array}{c}\text { Control } 2 \\
\text { (GFP) }\end{array}$ & 0 & $\begin{array}{c}1.83 \\
\pm \\
0.25\end{array}$ & & $1.83 \pm 0.25$ & & $\begin{array}{l}41.42 \\
\pm 18.4\end{array}$ & & $\begin{array}{c}41.42 \pm \\
18.4\end{array}$ & & 19 \\
\hline $\begin{array}{l}\text { Control3 (Not } \\
\text { electroporated }\end{array}$ & 0 & $\begin{array}{c}2.42 \\
\pm \\
0.23\end{array}$ & & $2.42 \pm 0.23$ & & $\begin{array}{r}54.86 \\
\pm 9.65\end{array}$ & & $54.86 \pm 9.65$ & & 121 \\
\hline $\begin{array}{l}\text { Control4 (Not } \\
\text { electroporated } \\
\text { ) }\end{array}$ & 0 & $\begin{array}{r}1.75 \\
\pm 0.1\end{array}$ & & $1.75 \pm 0.1$ & & $\begin{array}{c}44.12 \\
\pm \\
12.51\end{array}$ & & $\begin{array}{c}44.12 \pm \\
12.51\end{array}$ & & 51 \\
\hline
\end{tabular}




\section{$\underline{\text { Discussion }}$}

349 The VSCT is thought to provide peripheral and intrinsic spinal information to the cerebellum

350 in order to shape and update the output of spinal networks that execute motor behavior.

351 The lack of genetic accessibility to VSCT neurons hampers elucidation of their role in

352 locomotion. Using genetic toolbox to dissect the circuitry and manipulate neuronal activity

353 in the chick spinal cord, we studied spinal interneurons with VSCT characteristics. The main

354 findings in our study are that dl2 neurons in the chick lumbar spinal cord are commissural

355 neurons that innervate premotor neurons at the contralateral lumbar and brachial spinal

356 levels and granule neurons in the ipsilateral cerebellum. Hence, subpopulation of dI2 form a

357 part of the avian VSCT. Targeted silencing of dI2 neurons leads to impaired stepping in P8

358 hatchlings. We described the spatial distribution of sub-populations of dI2 neurons,

359 deciphered their connectomes, mapped the trajectory of their projection to the cerebellum,

360 and suggested possible mechanisms for the perturbed gait resulting from their genetic

361 silencing, as discussed below.

Using the intersection between genetic drivers and spatially restricted delivery of

363 reporters to define lumbar and brachial neurons, we have identified several targets of dI2

364 lumbar neurons. Ipsilateral lumbar dI2 neurons innervate contralateral lumbar dI2 neurons

365 as well as commissural and non-commissural lumbar pre-motoneurons. This connectivity

366 may affect the bilateral spinal output circuitry at the lumbar cord (e.g. (Bras et al., 1988,

367 Jankowska and Hammar, 2013)). Moreover, the ascending axons of lumbar dI2s, give off

368 grey matter collaterals innervating contralateral dI2s and commissural and non-commissural

369 pre-motoneurons throughout the brachial spinal cord (Fig. 6D). Therefore, lumbar dI2

370 neurons may also contribute to the inter-enlargement coupling described between the limb 
and wing moving segments of the spinal cord (e.g. (Valenzuela et al., 1990, Ruder et al.,

2016) for forelimb to hindlimb coupling connectivity in mice). base stepping, and variable limb movements during stepping. peripheral and intrinsic feedback to the cerebellum, leading to unreliable updating of the motor output produced by the locomotor networks, thereby impairing the bipedal stepping.

390 input from reticulospinal, rubrospinal and vestibulospinal pathways (Bras et al., 1988,

391 Jankowska and Hammar, 2013). Neurons from the lateral vestibular nucleus, have been

392 reported to innervate extensor motoneurons at the lumbar level, as well as interneurons

393 residing at the medial lamina VII (Murray et al., 2018), exactly at the location where dI2

394 neurons were found to reside in our study. Thus, the vestibulospinal tract may convey input 
395 directly to the ipsilateral motoneurons, and indirectly to contralateral motoneurons through

396 dI2 neurons that innervate contralateral pre-motoneurons. This way silencing of dI2 neurons

397 is expected to interfere with the descending regulation of the stability of the bipedal gait.

398 The local spinal connections between $\mathrm{dl} 2$ and the contralateral premotor neurons

399 and contralateral dI2, may serve an important component of coordinated limb movements.

400 d12 synapses were found on both ipsilateral and contralaterally projecting pre-

401 motorneurons, in their segmental level and in the brachial level, which regulates the

402 movement of the wings. Thus, dI2 neurons may affect the motor output of the contralateral

403 side of the cord, and also the ipsilateral side by contacting commissural pre-motorneurons.

404 In summary, our mapping studies of dI2 neurons and their connectomes followed by

405 characterization of the effects of their silencing on bipedal stepping, offer new insights on

406 the function of $d 12$ neurons in vertebrates. We suggest that lumbar $\mathrm{dl} 2$ neurons are not

407 merely used to relay sensory and intrinsic spinal networks information to the cerebellum,

408 but also act as active mediators of motor functions at the lumbar segments and at the wing

409 controlling brachial segments of the spinal cord. Further circuit deciphering studies of the

410 constituents of sub-populations of dI2s, their targets, and their descending inputs are

411 required to extend our understanding of the function of dI2 subpopulations in motor control

412 of movements. 


\section{Materials and Methods}

415

416

417 analysis.

\section{Animals}

Fertilized White Leghorn chicken eggs (Gil-Guy Farm, Israel) were incubated in standard conditions at $38^{\circ} \mathrm{C}$. All experiments involved with animals were conducted in accordance with the designated Experiments in Animals Ethic Committee policies and under its approval.

\section{D reconstruction and density plots analysis}

The codes for both 3D reconstruction and the density plots analysis were written in Matlab.

The density plots were generated based on cross section images transformed to a standard form. The background was subtracted, and the cells were filtered automatically based on their soma area or using a manual approach. Subsequently, two-dimensional kernel density estimation was obtained using the MATLAB function " $k d e 2 d$ ". Finally, unless indicated otherwise, a contour plot was drawn for density values between $20 \%$ and $80 \%$ of the estimated density range, in six contour lines.

\section{In-ovo electroporation}

A DNA solution of $5 \mathrm{mg} / \mathrm{mL}$ was injected into the lumen of the neural tube at $\mathrm{HH}$ stage 1718 (E2.75-E3). Electroporation was performed using $3 \times 50$ ms pulses at $25-30 \mathrm{~V}$, applied across the embryo using a 0.5-mm tungsten wire and a BTX electroporator (ECM 830). Following electroporation, $150-300 \mu \mathrm{L}$ of antibiotic solution, containing $100 \mathrm{unit} / \mathrm{mL}$ penicillin in Hank's Balanced Salt Solution (Biological Industry, Beit-Haemek) was added on top of the embryos. Embryos were incubated for 3-19 days prior to further treatment or

\section{Immunohistochemistry and In situ hybridization}


440 Embryos were fixed overnight at $4^{\circ} \mathrm{C}$ in $4 \%$ paraformaldehyde/0.1 $\mathrm{M}$ phosphate buffer,

441 washed twice with phosphate buffered saline (PBS), incubated in $30 \%$ sucrose/PBS for $24 \mathrm{~h}$,

442 and embedded in OCT (Scigen, Grandad, USA). Cryostat sections ( $20 \mu \mathrm{m}$ ). Sections were

443 collected on Superfrost Plus slides and kept at $-20^{\circ} \mathrm{C}$. For $100-\mu \mathrm{m}$ sections, spinal cords were

444 isolated from the fixed embryos and subsequently embedded in warm $5 \%$ agar (in PBS), and

$445100 \mu m$ sections (E12-E17) were cut with a Vibratome. Sections were collected in wells (free-

446 floating technique) and processed for immunolabeling.

447 The following primary antibodies were used-rabbit polyclonal GFP antibody 1:1000

448 (Molecular Probes, Eugene, Oregon, USA), mouse anti-GFP 1:100, Goat anti-GFP 1:300

449 (ABcam), rabbit anti-RFP 1:1000 (Acris), goat ChAT antibody 1:300 (Cemicon, Temecula, CA,

450 USA), mouse anti-synaptotagmin antibody 1:100 (ASV30), mouse anti-Lhx1/5 1:100 (4F2),

451 mouse anti-FoxP4 1:50 (hybridoma bank, University of lowa, lowa City, USA), mouse anti

452 Brn3a 1:50 (Mercury), rabbit anti-Pax2 antibody 1:50 (ABcam), chicken anti-lacZ antibody

453 1:300 (ABcam), rabbit anti Clabindin 1:200 (Swant), rabbit VGUT2 antibody (Synaptic

454 Systems, Göttingen, Germany), goat anti FoxP2 1:1000 (ABcam), goat anti FoxP1 1:100 (R\&D

455 Systems) and rabbit anti 5HT 1:100 (Immunostar). The following secondary antibodies were

456 used: Alexa Fluor 488/647-AffiniPure Donkey Anti mouse, rabbit and goat (Jackson) and

457 Rhodamin Red-X Donkey Anti mouse and rabbit (Jackson). Images were taken under a

458 microscope (Eclipse Ni; Nikon) with a digital camera (Zyla sCMOS; Andor) or a confocal

459 microscope (FV1000; Olympus).

460 In situ hybridization was performed as described (Avraham $\mathrm{O}$ et al., 2010). The following

461 probes were employed: Foxd3, vGlut2 and GAD1 probes were amplified from a cDNA of E6

462 chick embryo using the following primers. Foxd3: forward-TCATCACCATGGCCATCCTG and

463 Reverse -GCTGGGCTCGGATTTCACGAT. vGlut2: forward -GGAAGATGGGAAGCCCATGG and 
Reverse -GAAGTCGGCAATTTGTCCCC. GAD1: forward-TCTCACCTGGAGGAGCCATC and

reverse primers.

\section{AP staining}

469 The treated embryos were fixed with $4 \%$ paraformaldehyde-PBS for $24 \mathrm{~h}$ at $4^{\circ} \mathrm{C}$, and washed twice with PBS for $30 \mathrm{~min}$ at $4^{\circ} \mathrm{C}$. The fixed embryos were incubated at $65^{\circ} \mathrm{C}$ in PBS for 8 to

$47116 \mathrm{~h}$ to inactivate the endogenous AP activity. The treated embryos were washed with 100

$472 \mathrm{mM}$ Tris- $\mathrm{Cl}(\mathrm{pH}$ 9.5) containing $100 \mathrm{mM} \mathrm{NaCl}$ and $50 \mathrm{mM} \mathrm{MgCl}$, and the residual Placental

473 alkaline phosphatase activity was visualized by incubating the embryos with NBT/BCIP

474 (Roche) in the same buffer at $4^{\circ} \mathrm{C}$ for $24 \mathrm{~h}$. After extensively washing the embryos with PBS-

4755 mM EDTA, the spinal cord was imaged.

476

477

478

\section{Analysis of Left-right phase}

Stride duration was measured as the time from 'Right toe-off'/foot-off' to the next Right 'toe-off' (as a complete stride cycle for the right leg), and the 'half-cycle' duration as the time of Right-toe off to the time of Left toe off. the following formula was used to calculate the phase: ((LeftToeOff_1 - RightToeOff_1)/(RightToeOff_2-RightToeOff_1))*360.

\section{PRV infection}

We used two isogenic recombinants of an attenuated PRV strain Bartha (PRV Bartha) that express enhanced GFP (PRV152) and monomeric red fluorescent protein (PRV614). The viruses were harvested from Vero cell cultures at titers $4 \times 108,7 \times 108$ and $1 \times 109$ plaque forming units $(\mathrm{pfu} / \mathrm{mL})$, respectively. Viral stocks were stored at $-80^{\circ} \mathrm{C}$. Injections of $3 \mu \mathrm{L}$ of PRV152 or PRV614 were made into the thigh musculature of E13 or E14 chick embryos, using 
489

490

491

492

493

494

495

496

497

498

499

500

501

502

503

504

505

506

507

508

509

510

Hamilton syringe (Hamilton; Reno, NV, USA) equipped with a 33-gauge needle. The embryos were incubated for $36-40 \mathrm{~h}$ and sacrificed for analysis. For spinocerebellar projecting neurons labeling, we used a replication defective HSV (TK') that contains a lacZ reporter. The virus was injected in ovo into the cerebellum of E12-15 embryos, and the embryos were further incubated for 40-48. Alternatively, cholera toxin subunit B (CTB) conjugated to Alexa Fluor $^{\text {TM }} 647$ (ThermoFisher) was used for the same purpose, and was injected to the cerebellum of E12-15 embryos together with the virus for visualization of both cerebellar projecting neurons and upstream neurons.

\section{Force test}

The muscle strength was evaluated using the measurement of the angle of the fall from a ladder with a gradually increasing-angle. This test was repeated for each chicken at least 3 times, and the average falling angle was calculated.

\section{Behavioral tests and analysis}

The embryos were bilaterally electroporated, and were then allowed let to develop and hatch in a properly humidified and heated incubator. Afterwards, within 32 hours post hatching, the hatchling chicks were imprinted on the trainer. The P8 chicks were filmed in slow motion (240 fps) freely walking (side and top views). The following parameters were scored: 1) weight, 2) foot grip power, 3) kinematics parameters during overground locomotion: a) swing velocity, b) swing and stance duration, c) phase of footfalls, d) height of knee and tarsometatarso-phalangeal (TMP) joints, e) angles of the TMP and ankle joints, and f) stride width (distance between feet during the double stance phase). Using a semi-automated Matlab-based tracking software (Hedrick, 2008), several points of interest were encoded. The leg joints as well as the eye and the tail were tracked. 
514 The position of these reference points was used for computational analysis using an in-

515 house Matlab code for calculating different basic locomotion parameters (e.g. stick

516 diagrams, velocity, joints trajectory, angles, range, and elevation), steps pattern, and degree

517 of similarity (Haimson B et al. in preparation). Dunnett's test (Dunnett, 1955) was used to

518 perform multiple comparisons of group means following One-way ANOVA. Circular statistics

519 was used for analyses of angular data. Circular statistics was used for analyses of angular

520 data, utilizing Oriana (KCS, version 4). 
522 Acknowledgements: The authors thank Haya Falk for PRV purification; Alona Katzir, Cole

523 Bendor, Mevaseret Avital, Sapir Shevah, Eitan Yisraeli, Ruth Segal, Fedaa Bazan and Eden

524 Kimchi for technical assistance; Michael O'Donovan for comments on the manuscript. This

525 work was supported by grants to AK from the Israel Science Foundation (grant No. 1400/16),

526 The US-Israel Binational Science Foundation (grant No. 2017/172) and the Avraham and Ida

527 Baruch endowment fund.

528

529 Author contributions:

530 B.H., A.Klar and ALT designed research; B.H., and Y.H. preformed research; B.H., and M.D.

531 generated the kinematic analysis tools; Y.C. assisted in the post hatching analysis; A. Kania

532 provided reagents and comments on the research; B.H., A.Klar and ALT analyzed the data;

533 B.H., A.Klar and ALT wrote the paper.

534

535 Competing interests:

536 The authors declare no competing of interest. 
ALAYNICK, W. A., JESSELL, T. M. \& PFAFF, S. L. 2011. SnapShot: spinal cord development. Cell, 146, 178-178 e1.

ANDERSSON, L. S., LARHAMMAR, M., MEMIC, F., WOOTZ, H., SCHWOCHOW, D., RUBIN, C.-J., PATRA, K., ARNASON, T., WELLBRING, L., HJALM, G., IMSLAND, F., PETERSEN, J. L., MCCUE, M. E., MICKELSON, J. R., COTHRAN, G., AHITUV, N., ROEPSTORFF, L., MIKKO, S., VALLSTEDT, A., LINDGREN, G., ANDERSSON, L. \& KULLANDER, K. 2012. Mutations in DMRT3 affect locomotion in horses and spinal circuit function in mice. Nature, 488, 642-6.

AVRAHAM, O., HADAS, Y., VALD, L., ZISMAN, S., SCHEJTER, A., VISEL, A. \& KLAR, A. 2009. Transcriptional control of axonal guidance and sorting in dorsal interneurons by the Lim-HD proteins Lhx9 and Lhx1. Neural Dev, 4, 21.

BERMINGHAM, N. A., HASSAN, B. A., WANG, V. Y., FERNANDEZ, M., BANFI, S., BELLEN, H. J., FRITZSCH, B. \& ZOGHBI, H. Y. 2001. Proprioceptor pathway development is dependent on Math1. Neuron, 30, 411-22.

BIKOFF, J. B., GABITTO, M. I., RIVARD, A. F., DROBAC, E., MACHADO, T. A., MIRI, A., BRENNER-MORTON, S., FAMOJURE, E., DIAZ, C., ALVAREZ, F. J., MENTIS, G. Z. \& JESSELL, T. M. 2016. Spinal Inhibitory Interneuron Diversity Delineates Variant Motor Microcircuits. Cell, 165, 207-219.

BRAS, H., CAVALLARI, P. \& JANKOWSKA, E. 1988. Demonstration of initial axon collaterals of cells of origin of the ventral spinocerebellar tract in the cat. J Comp Neurol, 273, 584-92.

BUI, T. V., AKAY, T., LOUBANI, O., HNASKO, T. S., JESSELL, T. M. \& BROWNSTONE, R. M. 2013. Circuits for grasping: spinal dI3 interneurons mediate cutaneous control of motor behavior. NEURON, 78, 191-204.

CHENG, L., ARATA, A., MIZUGUCHI, R., QIAN, Y., KARUNARATNE, A., GRAY, P. A., ARATA, S., SHIRASAWA, S., BOUCHARD, M., LUO, P., CHEN, C. L., BUSSLINGER, M., GOULDING, M., ONIMARU, H. \& MA, Q. 2004. Tlx3 and Tlx1 are post-mitotic selector genes determining glutamatergic over GABAergic cell fates. Nat Neurosci, 7, 510-7.

DELILE, J., RAYON, T., MELCHIONDA, M., EDWARDS, A., BRISCOE, J. \& SAGNER, A. 2019. Single cell transcriptomics reveals spatial and temporal dynamics of gene expression in the developing mouse spinal cord. Development, 146.

DUNNETT, C. W. 1955. A Multiple Comparison Procedure for Comparing Several Treatments with a Control. Journal of the American Statistical Association, 50, 10961121.

FRANCIUS, C., HARRIS, A., RUCCHIN, V., HENDRICKS, T. J., STAM, F. J., BARBER, M., KUREK, D., GROSVELD, F. G., PIERANI, A., GOULDING, M. \& CLOTMAN, F. 2013. Identification of multiple subsets of ventral interneurons and differential distribution along the rostrocaudal axis of the developing spinal cord. PLoS One, 8, e70325.

FURUE, M., UCHIDA, S., SHINOZAKI, A., IMAGAWA, T., HOSAKA, Y. Z. \& UEHARA, M. 2010. Spinocerebellar projections from the cervical and lumbosacral enlargements in the chicken spinal cord. Brain Behav Evol, 76, 271-8.

FURUE, M., UCHIDA, S., SHINOZAKI, A., IMAGAWA, T., HOSAKA, Y. Z. \& UEHARA, M. 2011. Trajectories in the spinal cord and the mediolateral spread in the cerebellar cortex of spinocerebellar fibers from the unilateral lumbosacral enlargement in the chicken. Brain Behav Evol, 77, 45-54.

GOSGNACH, S., LANUZA, G. M., BUTT, S. J., SAUERESSIG, H., ZHANG, Y., VELASQUEZ, T., RIETHMACHER, D., CALLAWAY, E. M., KIEHN, O. \& 
GOULDING, M. 2006. V1 spinal neurons regulate the speed of vertebrate locomotor outputs. Nature, 440, 215-9.

GRIENER, A., ZHANG, W., KAO, H., WAGNER, C. \& GOSGNACH, S. 2015. Probing diversity within subpopulations of locomotor-related V0 interneurons. Dev Neurobiol, 75, 1189-203.

HADAS, Y., ETLIN, A., FALK, H., AVRAHAM, O., KOBILER, O., PANET, A., LEVTOV, A. \& KLAR, A. 2014. A 'tool box' for deciphering neuronal circuits in the developing chick spinal cord. Nucleic Acids Res, 42, e148.

HANTMAN, A. W. \& JESSELL, T. M. 2010. Clarke's column neurons as the focus of a corticospinal corollary circuit. Nat Neurosci, 13, 1233-9.

HEDRICK, T. L. 2008. Software techniques for two- and three-dimensional kinematic measurements of biological and biomimetic systems. Bioinspir Biomim, 3, 034001.

JANKOWSKA, E. \& HAMMAR, I. 2013. Interactions between spinal interneurons and ventral spinocerebellar tract neurons. J Physiol, 591, 5445-51.

JESSELL, T. M. 2000. Neuronal specification in the spinal cord: inductive signals and transcriptional codes. Nat Rev Genet, 1, 20-9.

JIANG, J., AZIM, E., EKEROT, C. F. \& ALSTERMARK, B. 2015. Direct and indirect spino-cerebellar pathways: shared ideas but different functions in motor control. Front Comput Neurosci, 9, 75.

LAI, H. C., SEAL, R. P. \& JOHNSON, J. E. 2016. Making sense out of spinal cord somatosensory development. Development, 143, 3434-3448.

MORIKAWA, Y., HISAOKA, T. \& SENBA, E. 2009. Characterization of Foxp2-expressing cells in the developing spinal cord. Neuroscience, 162, 1150-62.

MURRAY, A. J., CROCE, K., BELTON, T., AKAY, T. \& JESSELL, T. M. 2018. Balance Control Mediated by Vestibular Circuits Directing Limb Extension or Antagonist Muscle Co-activation. Cell Rep, 22, 1325-1338.

OSSEWARD, P. J., 2ND \& PFAFF, S. L. 2019. Cell type and circuit modules in the spinal cord. Curr Opin Neurobiol, 56, 175-184.

RUDER, L., TAKEOKA, A. \& ARBER, S. 2016. Long-Distance Descending Spinal Neurons Ensure Quadrupedal Locomotor Stability. Neuron, 92, 1063-1078.

SAKAI, N., INSOLERA, R., SILLITOE, R. V., SHI, S.-H. \& KAPRIELIAN, Z. 2012. Axon sorting within the spinal cord marginal zone via Robo-mediated inhibition of Ncadherin controls spinocerebellar tract formation. The Journal of neuroscience : the official journal of the Society for Neuroscience, 32, 15377-87.

SPANNE, A. \& JORNTELL, H. 2013. Processing of multi-dimensional sensorimotor information in the spinal and cerebellar neuronal circuitry: a new hypothesis. PLoS Comput Biol, 9, e1002979.

STECINA, K., FEDIRCHUK, B. \& HULTBORN, H. 2013. Information to cerebellum on spinal motor networks mediated by the dorsal spinocerebellar tract. J Physiol, 591, 5433-43.

SWEENEY, L. B., BIKOFF, J. B., GABITTO, M. I., BRENNER-MORTON, S., BAEK, M., YANG, J. H., TABAK, E. G., DASEN, J. S., KINTNER, C. R. \& JESSELL, T. M. 2018. Origin and Segmental Diversity of Spinal Inhibitory Interneurons. Neuron, 97, 341-355 e3.

UEHARA, M., AKITA, M., FURUE, M., SHINOZAKI, A. \& HOSAKA, Y. Z. 2012. Laterality of spinocerebellar neurons in the chicken spinal cord. J Vet Med Sci, 74, 495-8.

VALENZUELA, J. I., HASAN, S. J. \& STEEVES, J. D. 1990. Stimulation of the brainstem reticular formation evokes locomotor activity in embryonic chicken (in ovo). Brain Res Dev Brain Res, 56, 13-8. 
638 YUENGERT, R., HORI, K., KIBODEAUX, E. E., MCCLELLAN, J. X., MORALES, J. E., 639 HUANG, T. P., NEUL, J. L. \& LAI, H. C. 2015. Origin of a Non-Clarke's Column Division of the Dorsal Spinocerebellar Tract and the Role of Caudal Proprioceptive 641 Neurons in Motor Function. Cell Rep, 13, 1258-1271.

642 


\section{Figure legends}

645 Figure 1: Characterization and classification of dI2 neurons during embryonic development.

$646 \mathrm{~d} / 2$ interneurons were labeled by genetic intersection between Foxd3 and Ngn1 enhancers

647 (Avraham et al., 2009) (See Figure S1).

648 A-D. d12 axonal projection during development. At E5 (A) post mitotic dI2 neurons assume a

649 dorsolateral position and start to migrate ventrally. At E6 (B) dI2 neurons occupy the mid-

650 lateral domain. At E15-17 dI2 neurons are located at the medial lamina VII at the lumbar

651 level (LS3) (C) and the thoracic level (T1) (D). dI2 axons cross the floor plate (yellow

652 arrowheads), turn longitudinally at the ventral funiculus (white arrowheads) and eventually

653 elongate at the lateral funiculus (white arrows).

654 E. A graph indicating the fraction of dI2 neurons expressing TFs during development (based 655 on data from three E5, two E6 and two E14 embryos). F. Cross section of an E17 embryo at 656 the lumbar spinal cord (crural plexus level, LS2). Small-diameter dI2 neurons residing in 657 lamina VII ( $\left.F^{\prime}\right)$ and ventromedial large-diameter dI2 neurons in lamina VIII ( $\left.F^{\prime \prime}\right)$. G. Density 658 plots of dI2 somata in the sciatic plexus level (cyan, $N=374$ cells), dI 2 large (magenta) and $659 \mathrm{dI} 2_{\text {small }}$ (yellow) INs ( $N=33$ and $N=344$ cells, respectively, from 2 embryos).

660 See Figure S2 and S3.

661

662 Figure 2: dI2 neurons project to the cerebellum.

663 A. Experimental setup for labeling of cerebellar projecting dI2 neurons. dI2 neurons were 664 genetically targeted at $\mathrm{HH} 18$, and pre-cerebellar neurons were labeled using intra-cerebellar 665 injection of CTB or replication defective HSV-LacZ at E15. B. A cross section of E17 brainstem 666 and cerebellum. The dashed polygon in $B^{\prime}$ is magnified in B. dl2 axons reach the cerebellum, 667 enter into it via the superior cerebellar peduncle and cross the cerebellar midline. Calbindin 
668 (Purkinje neurons, magenta ( $\left.\mathrm{B}^{\prime}\right)$ or red (B)), synaptotagmin (yellow). C. A cross section of E17

669 cerebellar cortex. Lumbar-originating dI2 synapses (cyan) in the granular layer of the

670 anterior cerebellar cortex. Calbindin (Purkinje neurons, magenta), synaptotagmin (yellow).

671 D. A cross section of an E15 embryo at the lumbar spinal cord level (sciatic plexus level). Pre-

672 cerebellar neurons were infected and labeled by HSV-LacZ (magenta), and dI2 neurons express GFP (cyan). A large-diameter dI2 neuron co-expressing LacZ and GFP is indicated by

674 an arrow (magnification of the two channels in the insets). E. Density plots of dI2 and pre-

675 cerebellar neurons (density values $10-90 \%)$ in the sciatic plexus segments $(N=374$ and $N=289$

676 cells, respectively). F. CTB labeled pre-cerebellar neuron (magenta) is contacted by dI2

677 axonal terminals (cyan). G. Density plots of dI2 synapses and pre-cerebellar neuron somata

678 (density values $10-90 \%$ ) in the sciatic plexus segments ( $N=4735$ synapses and $N=289$ cells,

679 respectively). H,I. Quantification of the overlap in area and volume of the two density plots.

680 The plots are based on data from 3 embryos.

681 See Figure S4.

682

683 Figure 3: Synaptic inputs to dl2 neurons.

684 Schematic representations of the experimental design for labeling dI2::GFP or dI2::cherry INs

685 (cyan) and potential sources of synaptic inputs (yellow or magenta), supplemented by cell

686 soma density of dI2 INs and the synaptic densities are illustrated in A, B, C, D, E. The density

687 values presented are $10-80 \%, 20-80 \%, 25-80 \%, 30-50 \%$ and $20-80 \%$, respectively.

688 Examples of dI2 neurons contacted by axons or synaptic boutons are shown in $A^{\prime}, B^{\prime}, C^{\prime}$, and $D^{\prime}$; and their 3D reconstruction in $A^{\prime \prime}, B^{\prime \prime}, C^{\prime \prime}$, and $D^{\prime \prime}$. Genetic labeling was attained using 690 specific enhancers (Fig. S1) electroporated at $\mathrm{HH} 18$. 
691 A. DRG neurons form contacts on dI2 neurons. Inset in A: cross section of and E17 embryo at

692 the crural plexus level of the lumbar cord. A dorsally located dI2 neuron contacted by

693 numerous sensory afferents, magnified in $A^{\prime}$ and 3D-reconstructed in $A^{\prime \prime}$. ( $N=18$ sections)

694 B. Premotoneurons form contacts on dI2 neurons. dI2 neurons were labeled at HH18. At

695 E13, PRV virus was injected to the leg musculature, and the embryo was incubated until

696 premotoneuronal infection (39 hours) ( $N=34$ sections).

697 C. dl1 neurons form synapses on dI2 neurons. ( $N=8568$ synapses). C': A representative

698 SV2::cherry synapse on dI2 dendrite, positive for synaptotagmin. Demonstrated by a

699 horizontal and vertical optical sections in Z-axis (see cursors and color channels).

700 D. V1 neurons form synapses on dI2 neurons. ( $N=1923$ synapses).

701 E. $d 12$ neurons do not have 5 HT synaptic terminals ( $N=1718$ synapses). E17 cross sections of 702 dI2::GFP labeled embryos were stained for $5 \mathrm{HT}$.

703 F. Quantification of the overlap area of the different input sources and dI2 neuron densities 704 plots.

705 See Figure S5, S6.

707 Figure 4: Spinal synaptic targets of dI2 neurons.

708 A. A whole mount staining of spinal cord (thoracic segments) expressing alkaline

709 phosphatase (AP) in dI2 neurons. The lumbar dI2 neurons (not included in the image) were

710 labeled with AP. dI2 axon collaterals project and into the spinal cord (arrows).

711 B. Cross section of an E17 embryo at the crural plexus level of the lumbar cord. The axon

712 collaterals (white arrow) penetrating the gray matter of the contralateral side are evident.

713 Schematic representations of the experimental design for labeling synapses (dI2::SV2-GFP,

714 yellow) and potential targets (magenta) supplemented by cell soma density and dI2 synaptic 

boutons of dl2 neurons are shown in $C^{\prime}, D^{\prime}, E^{\prime}$, and $F^{\prime}$; and their 3D reconstruction in $C^{\prime \prime}, D^{\prime \prime}$,

$717 \mathrm{E}^{\prime \prime}$, and F". Genetic labeling was attained using dI2 enhancers (Fig. S1) electroporated at

$718 \mathrm{HH} 18$. Pre-MNs were labeled by injection of PRV-cherry into the hindlimbs (D, E) or the

719 forelimb (F) musculature, at E13. The embryos were incubated until pre-MNs infection (39

720 hours).

721 C. $d 12$ neurons innervate the contralateral dI2 neurons ( $N=4735$ synapses and $N=374$ cells,

722 respectively).

723 D. dI2 neurons innervate ipsilateral projecting premotoneurons at the sciatic plexus level

$724 \quad(N=4735$ synapses and $N=936$ cells, respectively).

725 E. dI2 neurons innervate contralateral projecting premotoneurons at the sciatic plexus level 726 ( $N=4735$ synapses and $N=47$ cells, respectively).

727 F. dI2 neurons innervate brachial ipsilateral projecting premotoneurons ( $N=2215$ synapses 728 and $N=286$ cells, respectively).

729 G. Quantification of the overlap area of different synaptic targets and dI 2 synapse density

730 plots, as the percentage of overlap between $\mathrm{d} / 2$ synapses and the target.

731 H. Quantification of the overlap in volume of the different synaptic targets and dI2 synapse 732 density plots, as the percentage of overlap between the synaptic target and dI2 synapses. 733 See Figure S7.

Figure 5: Kinematic analysis of locomotion in post-hatching chicks following neuronal

736 silencing of $\mathrm{d} / 2$.

737 A. Schematic illustration of chick hindlimb joints (bold) and bones (regular). The knee joint connects between the femur and the tibiotarsus, the ankle connects the tibiotarsus and the 
tarsometatarsus which connects to the phalanges by the tarsometatarso-phalangeal joint

740 (TMP). During the swing phase of birds, the ankle flexion leads to foot elevation, while the

741 knee is relatively stable.

742 B,C. Stick diagrams of stepping in a control chicken d2::GFP (B) and in a d2::TeTX chicken (C).

743 Arrows indicate falls and overshoots are denoted by arrowheads.

744 D. Overlays of knee height (demonstrated in insert) trajectories during the swing phase in all

745 analyzed steps of each of the control and TeTX treated P8 hatchlings are shown

746 superimposed with the respective $20 \%-80 \%$ color coded density plots as a function of the

747 percentage of swing (see Text and Materials and Methods). Arrows indicate falls and

748 overshoots are indicated by arrowheads.

749 E. Overlay of angular trajectories of the TMP joint (demonstrated in insert) during the swing

750 phase in all analyzed strides of each of the control and TeTX treated P8 hatchlings are shown

751 superimposed with the respective $20 \%-80 \%$ color coded density plots as a function of the

752 percentage of swing (see Text and Materials and Methods).

753

754 Figure 6. Increased inter-stride variability in TMP angle and knee height in TeTX-treated

755 chicks.

756 A. The percentage of steps with body collapses in the controls and TeTX manipulated

757 hatchlings ( $n=4$ and $n=5$, respectively). $p$-value $<0.0001$ (Z-test). See table 3 for single chick

758 fall proportions.

759 B. Analysis of the mean range of knee height changes during the swing phase of control and

760 TeTX treated chicks ( $n=4$ and $n=5$, respectively). $p$-value $<0.0001$ using a T-test allowing

761 different variances.

762 See Figure S8B and table 3 for single chick data and statistical analysis details. 
763 C. Analysis of the mean range of TMP angular excursions during the swing phase of control-

764 and TeTX-treated chicks. ( $n=4$ and $n=5$, respectively).

765 p-value $<0.0001$ Watson and Williams F test.

766 See Figure S8C and table 3 for single chick data and statistical analysis details.

767 D. Schematic illustration showing the connectome of lumbar dI2 neurons. dI2s (magenta)

768 receive synaptic input from sensory afferents (solid blue line indicates massive synaptic

769 input and dashed blue line indicates sparse innervation), Inhibitory and excitatory

770 premotoneurons (yellow), and from the contralateral lumbar dI2. dI2s innervate the

771 contralateral lumbar and brachial premotoneurons (both commissural and ipsilateral

772 projecting premotoneurons are innervated by $\mathrm{dl} 2$ ), the lumbar and brachial contralateral

$773 \mathrm{~d}$ I2, lumbar pre-cerebellar neurons (green) and the granule neurons in the cerebellum 
bioRxiv preprint doi: https://doi.org/10.1101/2020.01.07.898072; this version posted August 10, 2020. The copyright holder for this preprint (which was not certified by peer review) is the author/funder. All rights reserved. No reuse allowed without permission. 
Fig. 1
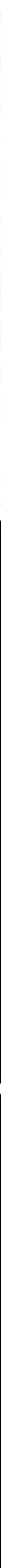
dl2::GFP
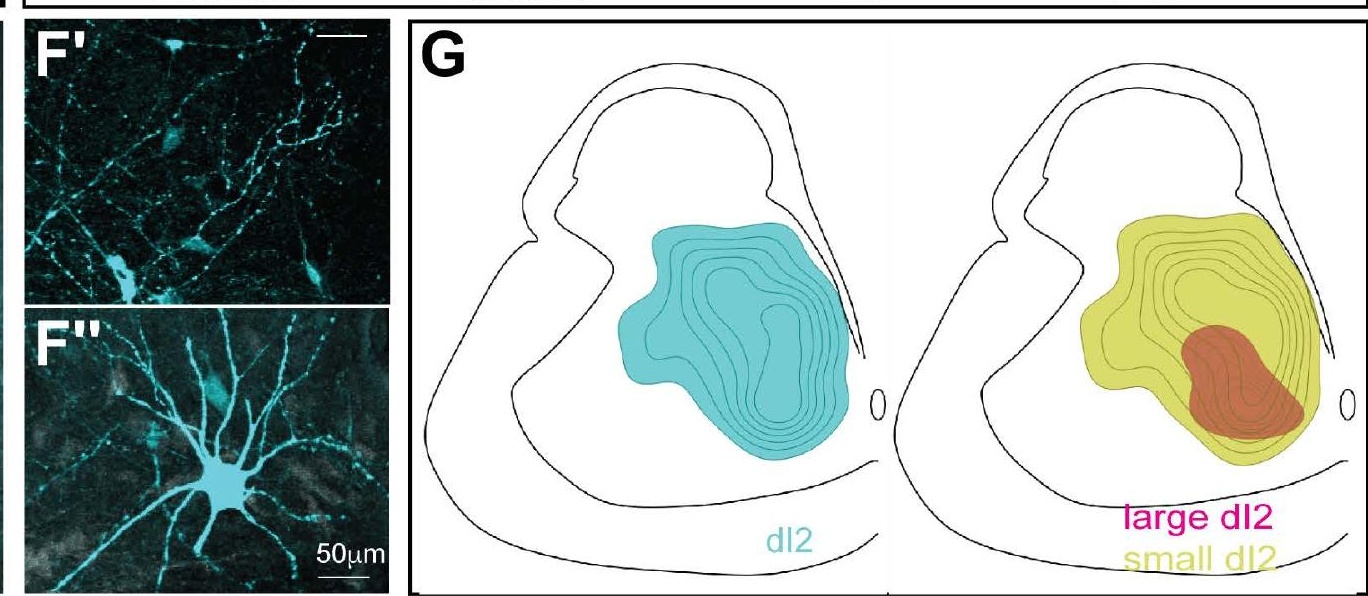
Fig. 2
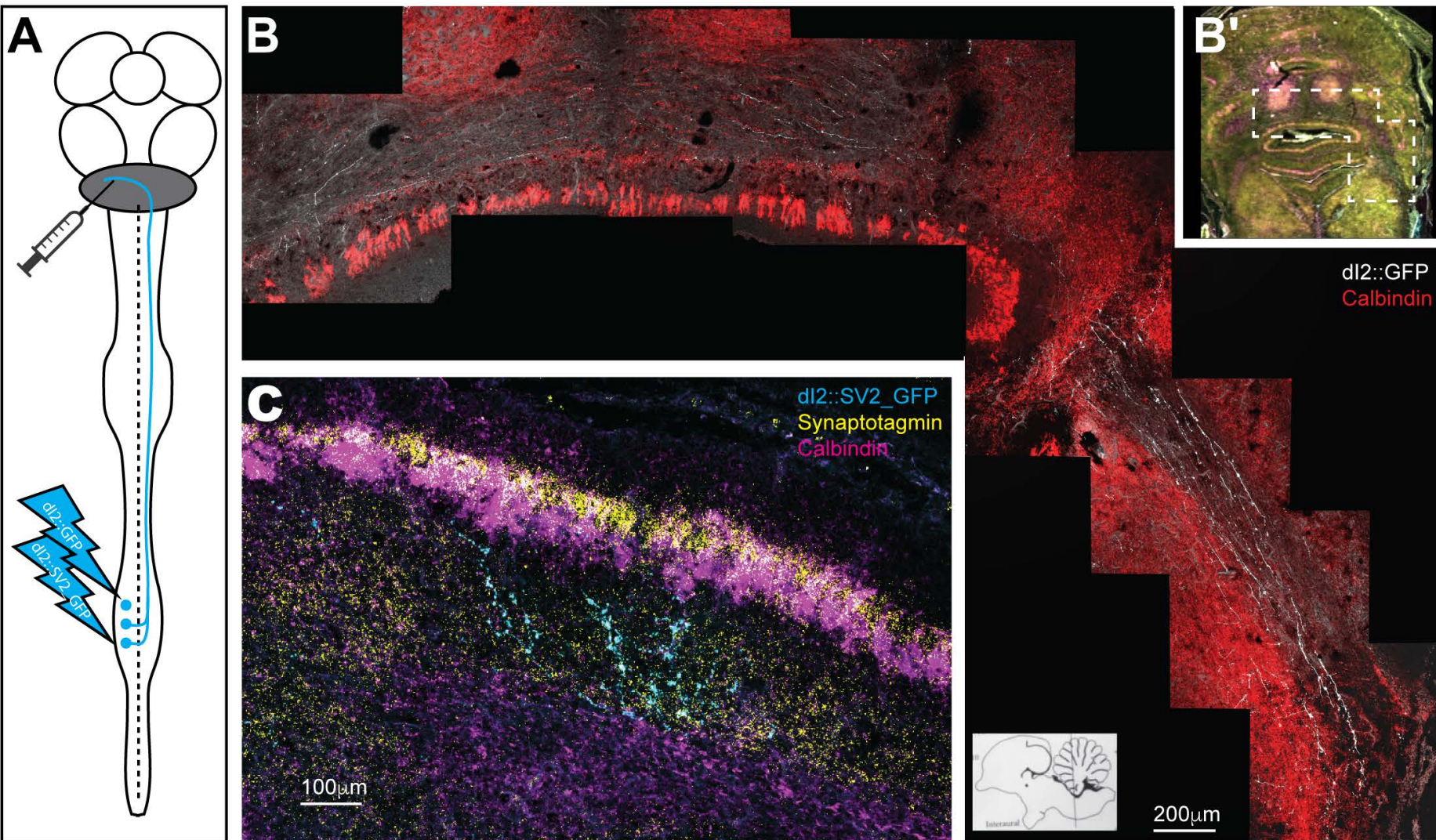

dl2::GFP

Calbindin
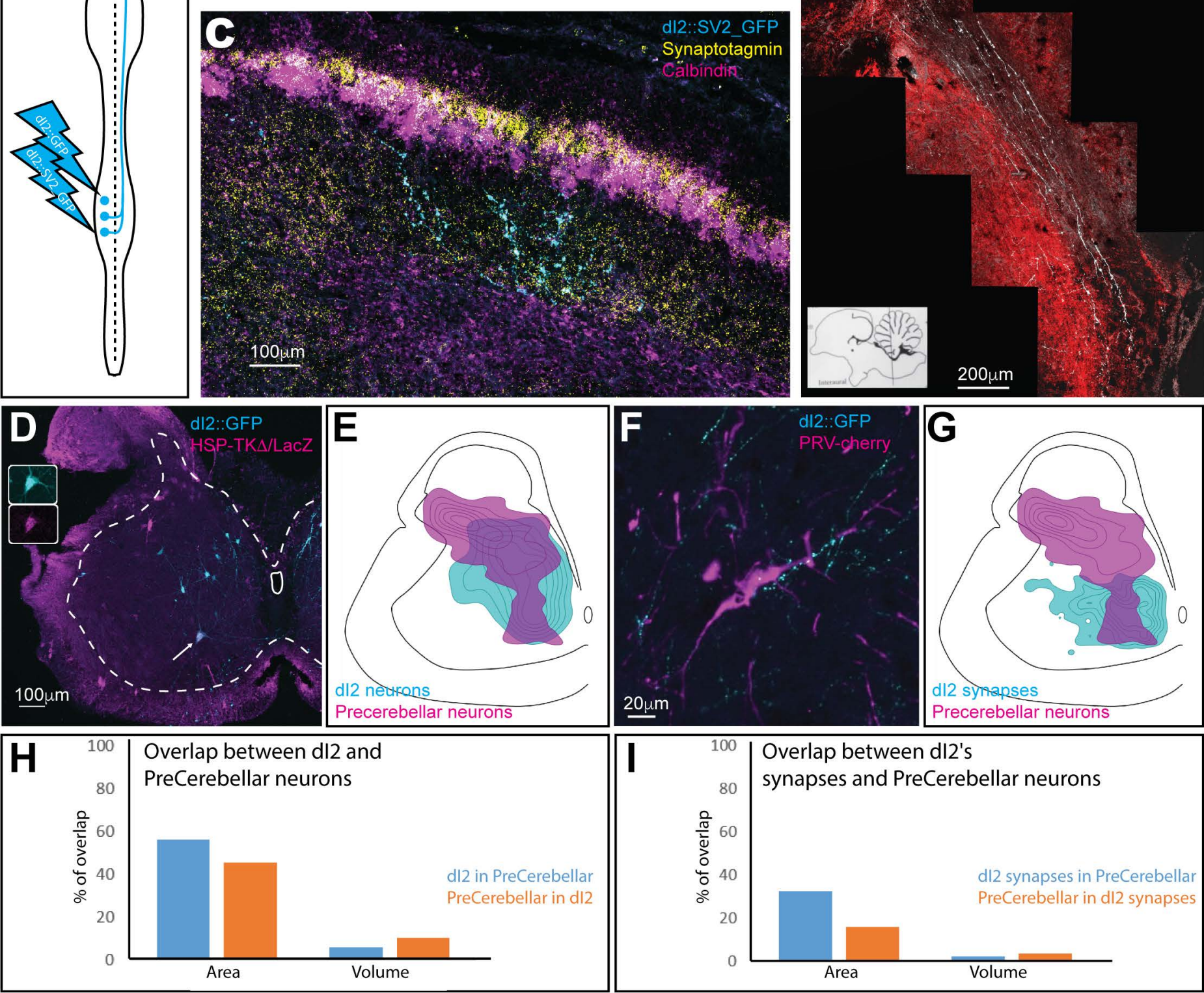

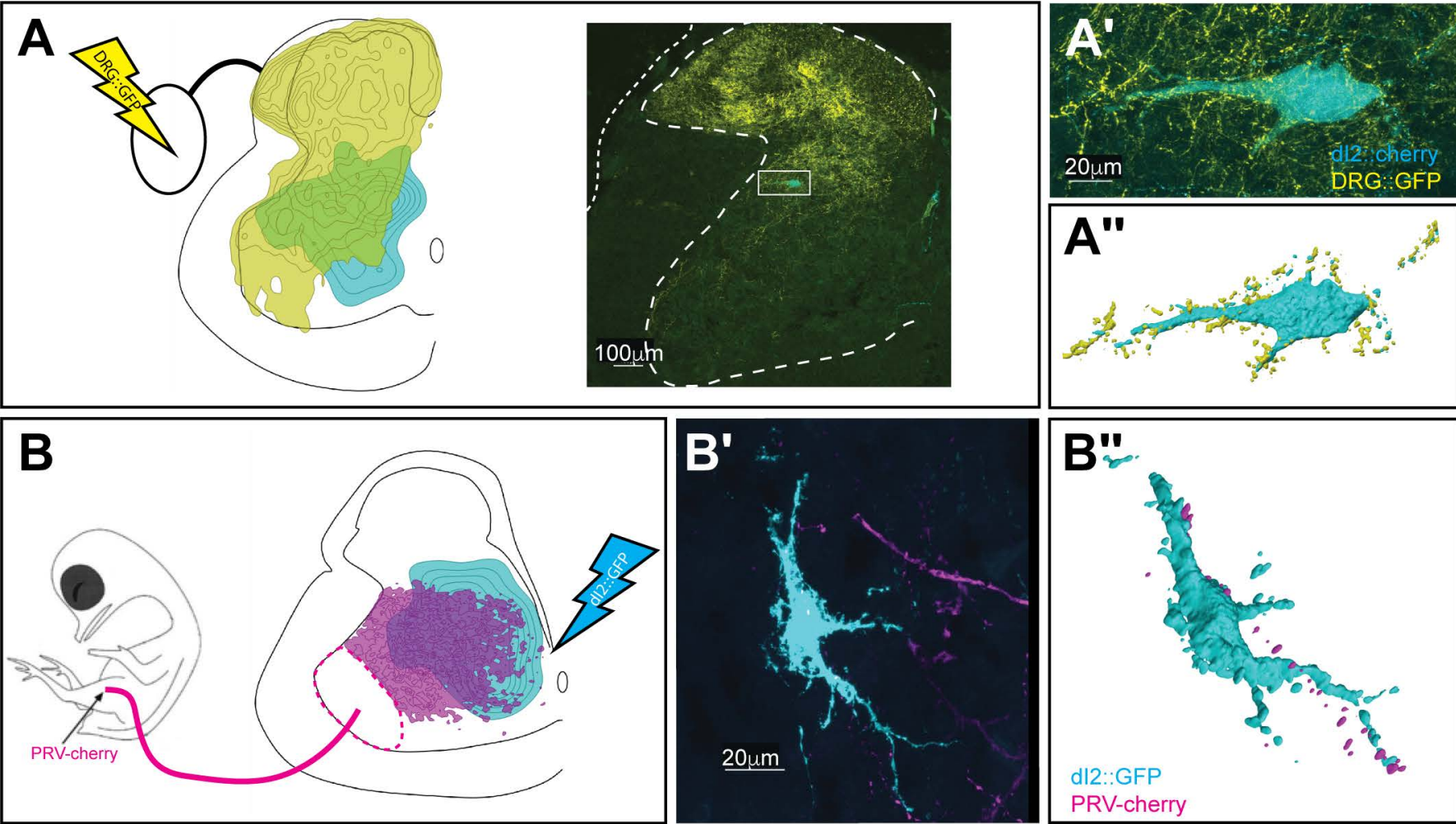

C
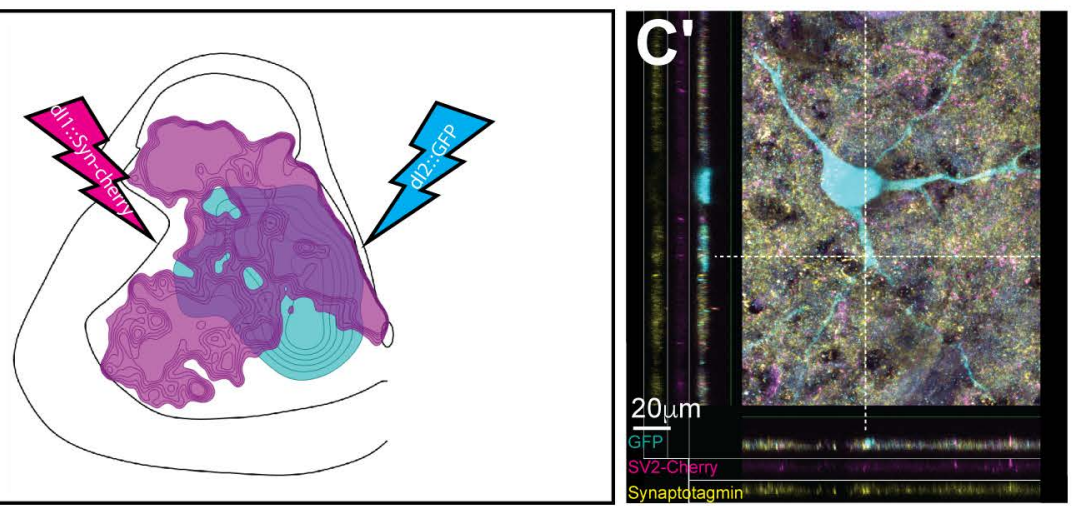

dI2::GFP
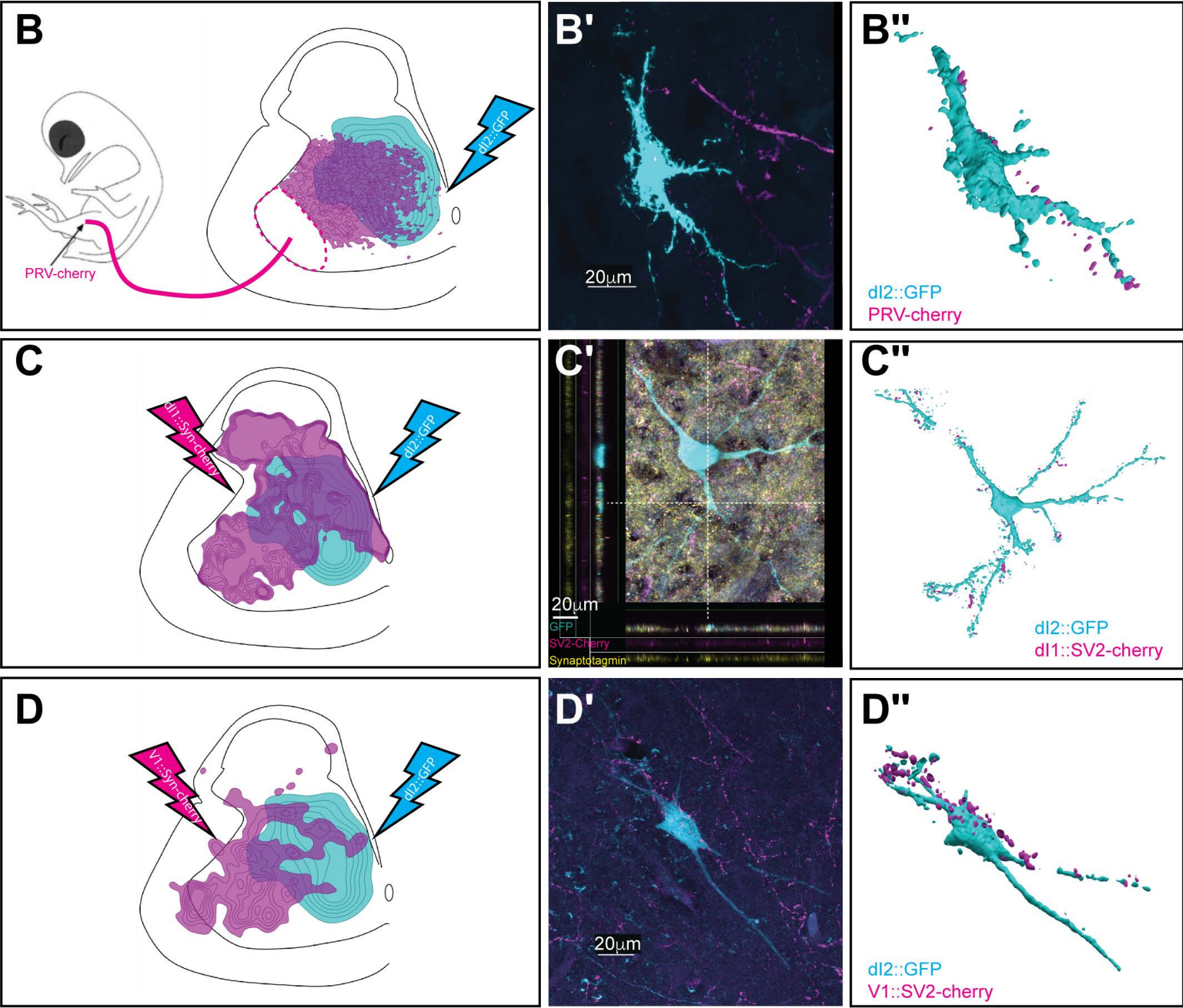

E

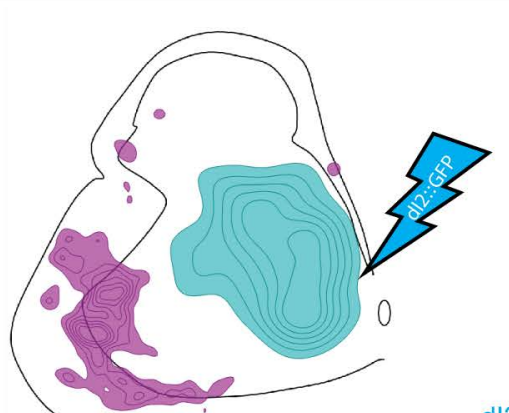

$\frac{\varkappa}{\frac{0}{0}} \mathbf{F}_{100} \quad$ colocalization of d 2 and synaptic inputs

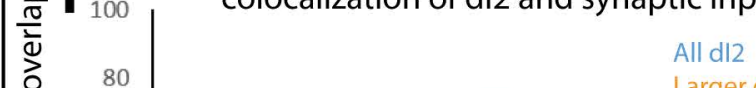

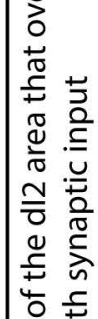


Fig. 6

A

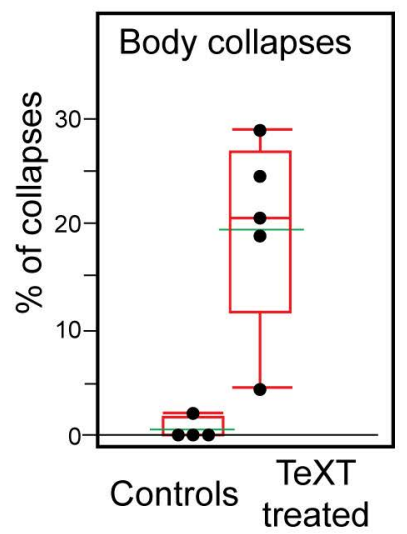

B

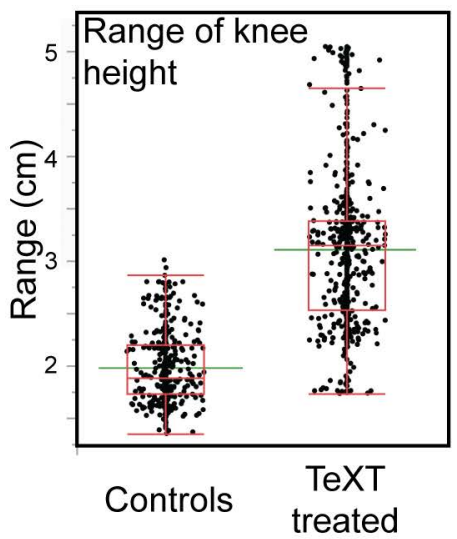

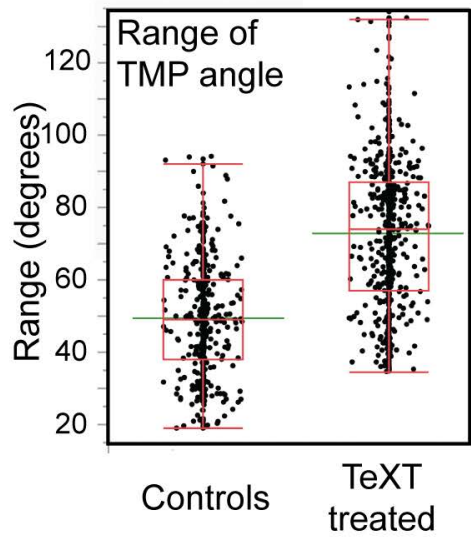

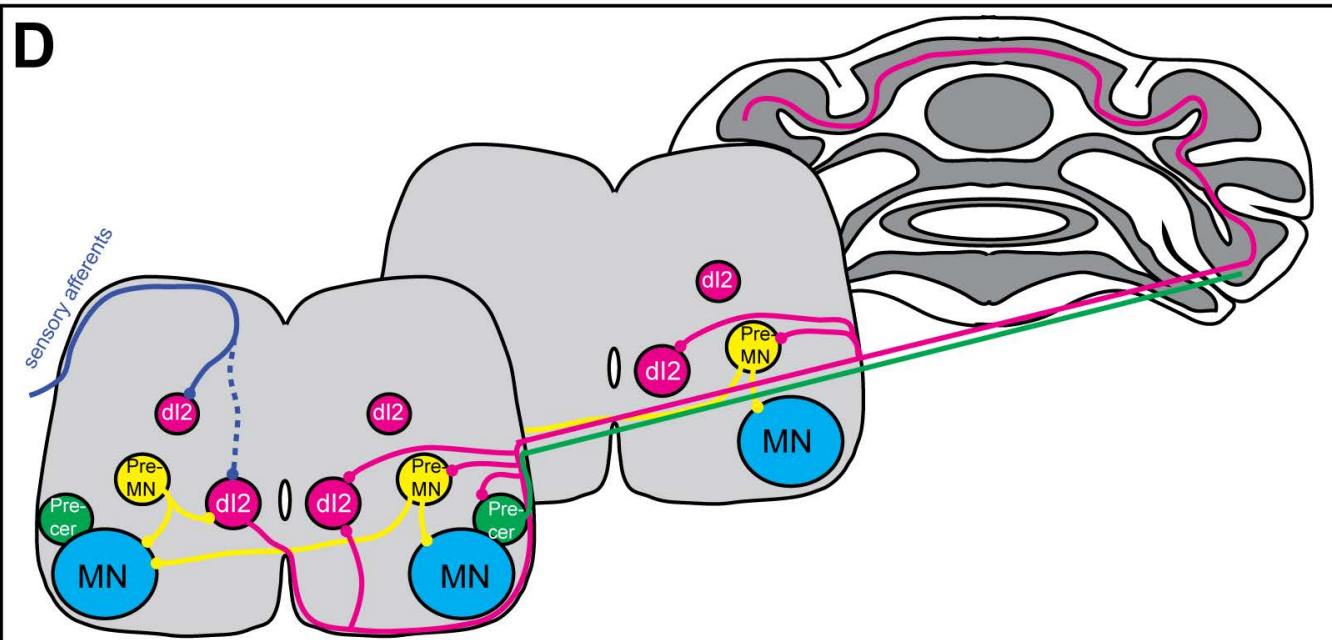

